\title{
Identification and Characterization of Novel Receptor-Interacting Serine/Threonine-Protein Kinase 2 Inhibitors Using Structural Similarity Analysis $\underline{\underline{[}}$
}

\author{
Mohamed Salla, Rodrigo Aguayo-Ortiz, Gaddafi I. Danmaliki, Alaa Zare, Ahmed Said, \\ Jack Moore, Vrajeshkumar Pandya, Robin Manaloor, Sunny Fong, Anna R. Blankstein, \\ Spencer B. Gibson, Laura Ramos Garcia, Pascal Meier, Khushwant S. Bhullar, \\ Basil P. Hubbard, Yahya Fiteh, Harissios Vliagoftis, Ing Swie Goping, Dion Brocks, \\ Peter Hwang, Carlos A. Velázquez-Martínez, and Shairaz Baksh
}

Departments of Biochemistry (M.S., G.I.D., A.S., J.M., V.P., I.S.G., P.H., S.B.), Pediatrics (A.Z., R.M., S.F., S.B.), Pharmacology (K.S.B., B.P.H.), Oncology (S.B.) Medicine (Y.F., H.V., P.H.), and Faculty of Pharmacy and Pharmaceutical Sciences (R.A.-O., D.B., C.A.-V.M.), University of Alberta, Edmonton, Alberta, Canada; Facultad de Química, Departamento de Farmacia, Universidad Nacional Autónoma de México, Mexico City, Mexico (R.A.-O.); Departments of Biochemistry and Medical Genetics and Immunology, University of Manitoba, Winnipeg, Manitoba, Canada (A.R.B., S.B.G.); Breakthrough Breast Cancer Research Center Chester Beatty Laboratories, London, United Kingdom (L.R.G., P.M.); Cancer Research Institute of Northern Alberta, Edmonton, Alberta, Canada (S.B.); and Women and Children's Health Research Institute, Edmonton, Alberta, Canada (S.B.)

Received December 13, 2017; accepted February 26, 2018

\section{ABSTRACT}

Receptor-interacting protein kinase 2 (RIP2 or RICK, herein referred to as RIPK2) is linked to the pathogen pathway that activates nuclear factor $\kappa$-light-chain-enhancer of activated $\mathrm{B}$ cells $\left(\mathrm{NF}_{\kappa} \mathrm{B}\right)$ and autophagic activation. Using molecular modeling (docking) and chemoinformatics analyses, we used the RIPK2/ponatinib crystal structure and searched in chemical databases for small molecules exerting binding interactions similar to those exerted by ponatinib. The identified RIPK2 inhibitors potently inhibited the proliferation of cancer cells by $>70 \%$ and also inhibited $\mathrm{NF} \kappa \mathrm{B}$ activity. More importantly, in vivo inhibition of intestinal and lung inflammation rodent models suggests effectiveness to resolve inflammation with low toxicity to the animals. Thus, our identified RIPK2 inhibitor may offer possible therapeutic control of inflammation in diseases such as inflammatory bowel disease, asthma, cystic fibrosis, primary sclerosing cholangitis, and pancreatitis.

\section{Introduction}

Activation of nuclear factor $\kappa$-light-chain-enhancer of activated $\mathrm{B}$ cells $(\mathrm{NF} \kappa \mathrm{B})$ proceeds through multiple pathways

The research in this study was supported by grants from Alberta Innovates - Health Solutions CRIO, Alberta Heritage Foundation for Medical Research, from the division of Hematology/Oncology/Palliative Care/ Epidemiology under Dr. David Eisenstat for funds to partially support this project and from the Department of Pediatrics/Stollery Children's Hospital-WCHRI Hair Massacure Donation Fund. Aguayo-Ortiz is very grateful to CONACyT (Mexico) for the fellowship granted (No. 510728/288862).

Authors M. Salla and R.Aguayo-Ortiz contributed equally to this manuscript.

https://doi.org/10.1124/jpet.117.247163.

S This article has supplemental material available at jpet.aspetjournals.org. involving tumor necrosis factor receptor 1 (TNF-R1) and pathogen-recognition receptors, such as Toll-like receptors (TLRs) (Orlowski and Baldwin, 2002; Madrid and Baldwin, 2003; Hayden and Ghosh, 2004) and nucleotide-binding oligomerization domain-containing protein 2 (NOD2), an intracellular pattern-recognition receptor. NOD2 is mainly stimulated by muramyl dipeptide (MDP), a bacterial product found in both Gram-positive and Gram-negative bacteria, and requires the obligate kinase, receptor-interacting protein kinase 2 (RIPK2), to promote both $\mathrm{NF} \kappa \mathrm{B}$ activation and an autophagic response (Tigno-Aranjuez et al., 2010). Mice with genetic deletion of Nod2/Ripk2 have a dysbiotic intestinal flora, resulting in altered susceptibility to intestinal

ABBREVIATIONS: 5-ROESY, rotating-frame overhauser enhancement spectroscopy; BAL, bronchoalveolar lavage; CRC, colorectal cancer; DMSO, dimethylsulfoxide; DSS, dextran sulfate sodium; IBD, inflammatory bowel disease; IL, interleukin; IP, immunoprecipitation; LPS, lipopolysaccharide; MDP, muramyl dipeptide; MolPort-016-359-762, 3-benzamido-4-methyl- $N$-[3-(1-methyl-1H-imidazol-2yl)phenyl]benzamide; MTT, (3-(4,5-dimethylthiazol-2-YI)-2,5-diphenyltetrazolium bromide); NF $\kappa$ B, nuclear factor $\kappa$-light-chain-enhancer of activated B cells; NOD2, nucleotide-binding oligomerization domain-containing protein 2; OD36, 6-Chloro-10,11,14,17-tetrahydro-13H-1,16-etheno-4,8-metheno-1Hpyrazolo[3,4-g][1,14,4,6]dioxadiazacyclohexadecine; OD38, 4,5,8,9-Tetrahydro-7H-2,17-etheno-10,14-metheno-1H-imidazo[1,5-g][1,4,6,7,12,14] oxapentaazacyclohexadecine; PDB, Protein Data Bank; RASSF1A, Ras association domain family protein 1A; RIPK2, receptor-interacting protein kinase 2; ROE, rotating-frame nuclear Overhauser enhancement; SIRT1, sirtuin 1; TLR, Toll-like receptor; TNF, tumor necrosis factor; WEHI-435, $\mathrm{N}$-(2-(4-amino-3-(p-tolyl)-1H-pyrazolo[3,4-d]pyrimidin-1-yl)-2-methylpropyl)isonicotinamide. 
inflammation (Ermann et al., 2014). In addition, the loss of Ripk2 has been demonstrated to result in the inability of cells to carry out mitophagy, leading to enhanced mitochondrial production of superoxide/reactive oxygen species and accumulation of damaged mitochondria that will trigger a caspase 1-dependent inflammasome activation (Lupfer et al., 2014). In the context of malignant transformation, knockdown of RIPK2 downregulated mRNA expression of E-cadherin and vimentin, both of which are involved in epithelial to mesenchymal transition and metastatic promotion (Wu et al., 2012). RIPK2 might thus play an important role in cell migration (Wu et al., 2012), metastasis (Wu et al., 2012), and offer alternate novel therapeutics for abnormal inflammation driven by the NOD2/RIPK2 pathway.

Most RIPK2 inhibitors were not designed to inhibit RIPK2, and thus, RIPK2 inhibition was an off-target effect of the use of these drugs. These included gefitinib/Iressa $\left(\mathrm{IC}_{50}\right.$ at $\left.50 \mathrm{nM}\right)$ (Canning et al., 2015), regorafenib, and other protein tyrosine kinases (Canning et al., 2015). Tigno-Aranjuez et al. (2014) reported on the identification of a novel class of RIPK2 inhibitors (OD36 [6-Chloro-10,11,14,17-tetrahydro-13H-1,16etheno-4,8-metheno-1H-pyrazolo[3,4-g] $[1,14,4,6]$ dioxadiazacyclohexadecine] and OD38 [4,5,8,9-Tetrahydro-7H-2,17etheno-10,14-metheno-1H-imidazo[1,5-g] $[1,4,6,7,12,14]$ oxapentaazacyclohexadecine]) that can inhibit $92 \%$ kinase activity of RIPK2 at $100 \mathrm{nM}$ and can alleviate inflammation in a rodent model for inflammatory bowel disease. However, like most RIPK2 inhibitors, at $100 \mathrm{nM},>80 \%$ inhibition of 10-FYN proto-ocogene, transforming growth factor 2 beta, activin receptor like kinase 2 , and lymphocyte specific protein tyrosine kinase and Lck was also observed. WEHI435 , a recently characterized small-molecule RIPK2 inhibitor, was demonstrated to delay RIPK2 ubiquitination and $\mathrm{NF}_{\kappa} \mathrm{B}$ activation downstream of NOD2 activation. In addition, WEHI-435 (N-(2-(4-amino-3-(p-tolyl)-1H-pyrazolo [3,4-d]pyrimidin-1-yl)-2-methylpropyl)isonicotinamide) interfered with cytokine production in vitro and in vivo and ameliorated experimental autoimmune encephalomyelitis in mice (Nachbur et al., 2015). Recently, GlaxoSmithKline isolated an RIPK2 inhibitor, GSK-583 (6-(tert-butylsulfonyl)$\mathrm{N}$-(5-fluoro-1H-indazol-3-yl)quinolin-4-amine), based on using the full RIPK2 protein to screen a DNA-encoded library collection with a fluorescence polarization-based binding assay as a readout (Haile et al., 2016). This bound to the ATP binding pocket of the kinase domain and inhibited RIPK2 with an $\mathrm{IC}_{50}$ of $5-50 \mathrm{nM}$, depending on the assay. Last, Novartis recently reported on their RIPK2 inhibitor, obtained by virtual screening of their proprietary library, that can inhibit RIPK2 with an $\mathrm{IC}_{50}$ of $3 \mathrm{nM}$ (He et al., 2017). No in vivo applicability was analyzed, but it can selectively inhibit MDPpromoted cytokine production in peripheral blood mononuclear cells and bone marrow-derived mouse macrophages. A kinome analysis was not carried out, and thus, it is unknown if significant off-target effects exist in the presence of the Novartis RIPK2 inhibitor.

Because of robust off-target effects of most RIPK2 inhibitors, we carried out molecular modeling (docking) and cheminformatics analyses by carefully analyzing the only known crystal structure of RIPK2 in association with the c-ABL kinase inhibitor ponatinib (https://www.rcsb.org/pdb/explore/ macroMoleculeData.do? structure $I d=4 \mathrm{C} 8 \mathrm{~B}$ ). Utilizing this structure, we searched chemical databases for scaffolds exerting significant binding interactions similar to (or stronger than) those exerted by ponatinib, determined by comparing their free energies when docked inside of the RIPK2 binding site. In addition to RIPK2 binding as part of this computer-based (initial) screening, we selected compounds that inhibited epidermal growth factor receptor $\left(\mathrm{IC}_{50}\right.$ values $>1000 \mathrm{nM}$; weak inhibitory activity) along with weak binding interactions in the EGFR and c-ABL binding sites, two common off-targets for previously identified RIPK2 inhibitors. Several hits are shown in Fig. 1 and Table 1, and these compounds have unknown biologic features. Since RIPK2 signals downstream of NOD2 and is linked to $\mathrm{NF} \kappa \mathrm{B}$ and autophagy activation, we speculated that the in vivo use of our selective RIPK2 inhibitors would target $\mathrm{NF}_{\kappa} \mathrm{B}$ activation and possibly resolve abnormal inflammation states.

\section{Methods}

Additional methods can be found in the Supplemental Experimental Procedures online.

Pharmacophore Search. Commercially available subsets of the ZINC (15,868,179 compounds) and MolPort (7,241,662 compounds) databases were screened using the lead drug ponatinib as a reference. We used the ZINCPharmer pharmacophore search server (Koes and Camacho, 2012), and pharmacophore features were identified directly from the RIPK2 structure [Protein Data Bank (PDB) identifier: 4C8B] and used for the screening. In this regard, a total of 74 compounds were selected from both databases. The selected compounds were then docked into the RIPK2 binding site.

Structure Similarity Search. The PubChem (Kim et al., 2016) compound library (more than 80 million molecules) was compared with ponatinib to filter all molecules which are at least $60 \%$ similar to this compound (10,655,787 molecules). We selected the most similar ( $\geq 90 \%$; 803 compounds) and less similar $(<62 \%$; 50,000 compounds randomly selected from a total of $2,607,266)$ structures using the PubChem Fingerprint implemented in the ChemmineR package (Bioconductor, Seattle, WA) (Cao et al., 2008) of the R program (R Development Group, Vienna, Austria). The filtered compounds were then subjected to another filtering step based on Lipinski's rule of five (Lipinski et al., 2001), removing the duplicates and inorganic molecules with the FAFDrugs3 server (Lagorce et al., 2015) $(22,274$ compounds). Finally, a total of 3000 compounds were selected and optimized using the obminimize tool of the OpenBabel toolbox (Open Babel, Pittsburgh, PA) (O'Boyle et al., 2011) for the docking studies.

Molecular Docking. The crystal structure of the human RIPK2 (PDB: 4C8B) (https://www.rcsb.org/pdb/explore/macroMoleculeData. do? structureId=4C8B) was retrieved from the PDB (Bernstein et al., 1977). Some residues in the three-dimensional structures were missing, and consequently, these side-chain atoms were added using the WHAT IF web interface (Vriend, 1990). The protein was subjected to an energy minimization using the AMBER99SB force field by 1000 step steepest-descent minimization followed by 100-conjugate gradient minimization using University of California, San Francisco CHIMERA version 1.9 software (University of California, San Francisco, CA) (Pettersen et al., 2004). Polar hydrogens, Gasteiger-Marsili empirical atomic partial charges, and the AutoDock atom types were computed using the MGLTools version 1.5.4 package (Molecular Graphics Laboratory at The Scripps Research Institute, La Jolla, CA) (Morris et al., 2009). The torsional root and branches of the ligands were chosen with the same program, allowing flexibility for all rotational bonds (except for the amide bond). In addition, the software was used to assign Gasteiger-Marsili atomic charges to all ligands (Gasteiger et al., 1978). Docking calculations were performed using the AutoDock Vina software (Molecular Graphics Lab at The Scripps Research Institute) (Trott and Olson, 2010). A grid box of $28 \AA^{3}$ and centered at the binding site was used to calculate the atom types 

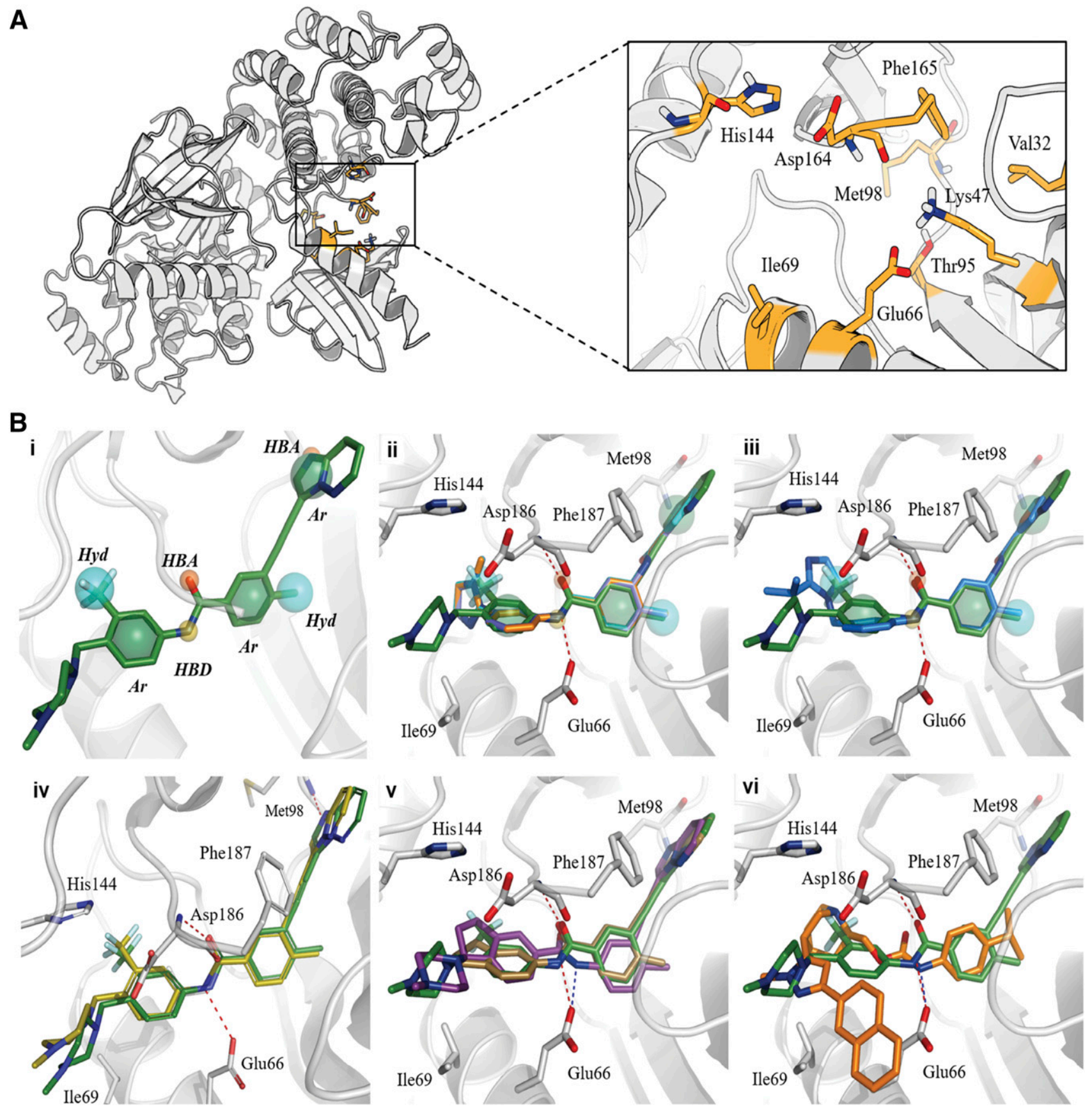

Fig. 1. (A) Schematic of the RIPK2 active site. (B) Representation of pharmacophore points: hydrogen bond donor (HBD, yellow), hydrogen bond acceptor (HBA, orange), hydrophobic (Hyd, cyan) and aromatic (Ar, green). Predicted binding modes of ponatinib (green) (i), MolPort-016-359-762 (orange) (ii), and MolPort-016-412-727 (blue) (iii) in the RIPK2 binding site. (iv) Comparison between the binding position of ponatinib found within the crystal structure (yellow) and the binding mode predicted by AutoDock Vina (green). Predicted binding modes of PC_57410628 (purple), PC_68349611 (brown) (v), and PC_44716361 (MolPort-001-746-327, orange) (vi) in the RIPK2 binding site. All hydrogens were omitted for clarity. The dotted lines represent distances for the formation of hydrogen bonds.

needed for the calculation. A total of nine runs were performed with an exhaustiveness of 50. The best binding mode of each molecule was selected based on the lowest binding free energy score. The threedimensional figures were generated using PyMOL Molecular Graphics System (DeLano Scientific LLC, Palo Alto, CA).

RIPK2 In Vitro Kinase Assay. For this protocol, samples were lysed in $1 \times$ RIPK2 lysis buffer [50 mM Tris ( $\mathrm{pH} 7.5$ ), $0 \mathrm{mM}$ $\mathrm{MgCl}_{2}, 1 \%$ Triton X-100, $1 \mathrm{mM}$ dithiothreitol, $1 \mathrm{mM}$ EDTA, $1 \mathrm{mM}$ EGTA, with freshly added $1 \mathrm{mM} \beta$-glycerophosphate and protease inhibitors (phenylmethylsulfonyl fluoride and aprotinin)] and immunoprecipitated overnight using $1.5 \mu \mathrm{g}$ rabbit anti-RIPK2 antibody. The next day, protein $\mathrm{G}$ sepharose was used to immunoprecipitate the RIPK2 protein complex immunoprecipitation (IP) for 1.5 hours. Following this incubation, samples were washed once in $1 \times$ kinase wash buffer [50 mM Tris (pH 7.5), $150 \mathrm{mM} \mathrm{NaCl}, 1 \%$ Triton X-100, 1 mM EDTA] followed by two washes with $1 \times$ kinase buffer

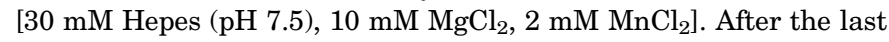
wash, $20 \mu \mathrm{l}$ of water was added to beads, followed by $1 \times$ kinase buffer and ${ }^{32} \mathrm{P}-\gamma$-ATP, and kinase reaction was allowed to proceed for $45-60$ minutes at $30^{\circ} \mathrm{C}$. Protein loading dye was added to the mix, 
TABLE 1

Calculated binding free energies of potential RIPK2 inhibitors

ZINC, MolPort, and PubChem (PC) molecule identification numbers are shown. MolPort-016-359-762 is RIPK2 inhibitor 1 and PC_44716361 (MolPort-001-746-327) is RIPK2 inhibitor 2.

\begin{tabular}{lcllc}
\hline Compound & $\frac{\Delta \mathrm{G}_{\text {bind }}}{n}$ & & Compound & $\frac{\Delta \mathrm{G}_{\text {bind }}}{k c a l / m o l}$ \\
& & & $k c a l / m o l$ \\
\hline Pharmacophore & -11.9 & MolPort-015-604-588 & -10.6 \\
Ponatinib & -11.5 & MolPort-016-412-727 & -10.6 \\
MolPort-016-359-762 & & -10.1 \\
MolPort-015-752-252 & -10.8 & ZINC02739307 & \\
Structure similarity & & & -11.5 \\
PC_57410628 & -12.0 & PC_58945682 & -11.5 \\
PC_44716361 & -118 & PC_58945685 & -11.5 \\
PC_24826801 & -118 & PC_68349611 & -11.3 \\
PC_57405602 & -11.6 & PC_58945669 & -11.1 \\
PC_58945635 & -11.6 & PC_40780119 &
\end{tabular}

and samples were boiled and separated on an SDS-PAGE gel. The gel was dried and then exposed to X-ray film to capture the autophosphorylation of RIPK2.

NFкB Gene Reporter Luciferase Assay. The Dual-Luciferase Reporter Assay System (E1910; Promega, Madison, WI) was used to perform dual-reporter assays on $\mathrm{NF}_{\kappa} \mathrm{B}$ luciferase and Renilla luciferase (internal control). In brief, HCT116 colon cancer cells were equally seeded at a density of $3 \times 10^{4}$ in six-well plates and allowed to attach for 24 hours. Prior to transfection, cells were washed with serum-free medium three times. Dual transfection was carried out using $12 \mu \mathrm{l}$ of polyethylenimine to $3 \mu \mathrm{g}$ of $\mathrm{NF} \kappa \mathrm{B}$ luciferase construct and $60 \mathrm{ng}$ of Renilla luciferase construct. Twenty-four hours after transfection, cells were treated with the different drugs for 24-36 hours. Cells were then lysed using the passive lysis buffer provided by the kit for 30 minutes on ice. Lysate was spun down for 8 minutes at 10,000 rpm, and $20 \mu \mathrm{l}$ of cell lysate was transferred into a 96 -well plate. Luciferase assays were analyzed based on the ratio of Firefly/Renilla to normalize cell number and transfection efficiency.

Intestinal Inflammation Injury Model. Animals were administered $3 \%(\mathrm{w} / \mathrm{v})$ dextran sulfate sodium (DSS; molecular weight of 40,000-50,000; MP Biomedicals, Santa Ana, CA) in the drinking water for 7 days followed by recovery for 7 days. They were monitored for piloerection, bloatedness, tremors, lack of movement, rectal bleeding, and weight loss [all on a scale of $0-5$, with 5 being very severe; adapted from Madsen et al. (2001)]. Animals were euthanized once rectal bleeding became grossly apparent. For weight loss, the following scores were assigned: 0 for no weight loss, 1 if $5 \%$ loss, 2 for $5 \%-10 \%$ loss, 3 for 10\%-15\% loss, 4 for 15\%-20\% loss, and 5 for $>20 \%$ loss in initial body weight. Disease activity indices were the sum of all individual scores. All animals were male of the C57BL/6 background and at 10-12 weeks of age or $25 \mathrm{~g}$ in body weight at the beginning of the experiment.

Lung Inflammation Model. Male Balb/c mice (6-8 weeks) were sensitized on days 1 and 6 via intraperitoneal injection of $0.9 \%$ sterile saline $(0.5 \mathrm{ml})$ or $0.9 \%$ saline containing $10 \mu \mathrm{g}$ of ovalbumin and $2 \mathrm{mg}$ of $\mathrm{Al}(\mathrm{OH})_{3}$. After light anesthesia with ketamine $(75 \mathrm{mg} / \mathrm{kg})$ and acepromazine $(2.5 \mathrm{mg} / \mathrm{kg})$, mice were challenged intranasally with $25 \mu \mathrm{l}$ of saline containing $50 \mu \mathrm{g}$ of ovalbumin or saline alone as a control on days 12 and 14. RIPK2 inhibitor 1 ( $1 \mu \mathrm{g} / \mathrm{g}$ body weight) or $30 \%$ dimethylsulfoxide (DMSO; solvent control for drug) was injected intraperitoneally on days 12,13 , and 14 (on days 12 and 14 , the injection was performed 1 hour before the intranasal challenge with ovalbumin). On day 15, mice were euthanized with an intraperitoneal injection of $2 \mathrm{mg}$ of sodium pentobarbital. Cardiac puncture was used to collect blood, followed by tracheal intubation with polyethylene tubing. The lungs were washed twice, with $1 \mathrm{ml}$ of phosphate-buffered

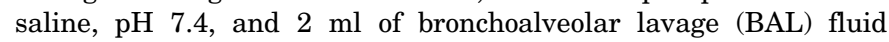
was collected. BAL fluid was spun at $300 \mathrm{~g}$ for 5 minutes. Cell pellets were resuspended in phosphate-buffered saline, total cell count was performed, and then cytospins were prepared with 5000 cells. The slides were stained with Diff-Quick (Polysciences, Warrington, PA), and differential cell counts were performed to assess allergic airway inflammation. This was done in collaboration with Dr. Harissios Vliagoftis (University of Alberta, Edmonton, AB, Canada).

Mass Spectrometry. Direct-infusion measurements were carried out on an LTQ Orbitrap XL mass spectrometer (Thermo Fisher Scientific, Waltham, MA) using the Ion Max ESI source (Thermo Fisher Scientific). The on-board syringe pump was used with a $100-\mu \mathrm{l}$ Hamilton syringe and a flow rate of $5 \mu \mathrm{l} / \mathrm{min}$. The following parameters were used: sheath gas flow of 15 arbitrary units, auxiliary gas flow of 5 arbitrary units, spray voltage of $3.5 \mathrm{kV}$, tube lens voltage of $110 \mathrm{~V}$, capillary temperature of $275^{\circ} \mathrm{C}$, capillary voltage of $35 \mathrm{~V}$, and capillary temperature of $275^{\circ} \mathrm{C}$. The automatic gain control target was set to $1 \mathrm{e}^{6}$ and the maximum injection time to $200 \mathrm{~ms}$. The resolution was set at 100,000, and two microscans were recorded.

NMR Spectroscopy. NMR experiments were run on a Varian Inova $500 \mathrm{MHz}$ spectrometer (Agilent, Santa Clara, CA) at $30^{\circ} \mathrm{C}$. The proton chemical shifts were measured relative to the residual protonated methyl $\left(\mathrm{CHD}_{2}\right)$ signal of $d_{6}$-DMSO (2.50 ppm); coupling constants $(J)$ are reported in $\mathrm{Hz}$. Standard notation is used to describe the multiplicity of signals observed in ${ }^{1} \mathrm{H}$ NMR spectra: singlet (s), doublet (d), triplet (t), broad (b), etc. Through-space and through-bond connectivities were observed using a two-dimensional ${ }^{1} \mathrm{H}^{-1} \mathrm{H}$ rotatingframe Overhauser enhancement spectroscopy $\left({ }^{1} \mathrm{H}^{-1} \mathrm{H}\right.$ ROESY) experiment (mixing time $200 \mathrm{~ms}$ ). One-dimensional experiments were processed using VNMRJ (Varian Associates, Palo Alto, CA), and two-dimensional ${ }^{1} \mathrm{H}-{ }^{1} \mathrm{H}$ ROESY was processed using NMRPipe/NMRDraw software (Ad Bax group, Bethesda, MD). RIPK2 inhibitor 1 was prepared by dissolving $0.3 \mathrm{mg}$ in $500 \mu \mathrm{l} d_{6}$-DMSO. RIPK2 inhibitor 2 was prepared by dissolving $0.3 \mathrm{mg}$ in $500 \mu \mathrm{l}$ of $d_{6}$-DMSO (Delaglio et al., 1995).

Use of Animals. All animal experimentation was performed in accordance with the University of Alberta institutional guidelines as defined by the Animal Care and Use Committee.

Statistical Analysis. Statistical analysis was carried out by analysis of variance and Student's $t$ test (two-tailed) as indicated.

\section{Results}

Pharmacophore Search. The ponatinib/RIPK2 complex (PDB: 4C8B) has revealed significant binding interactions of this inhibitor in the RIPK2 active site. Based on this crystal structure, the active site is localized in a binding pocket formed by at least eight different amino acid residues, including Val32, Lys47, Glu66, Ile69, Leu70, Thr95, Met98, and Asp164 (Fig. 1A). In this work, the ZINC and MolPort databases were screened with the ZINCPharmer pharmacophore search server using the lead drug Ponatinib as a query. Several pharmacophoric features were identified directly from the RIPK2 structure and used for the screening. In this regard, a total of 74 compounds were identified from both databases with pharmacophoric points similar to ponatinib (Fig. 1B). The compounds were then docked into the same RIPK2 binding site in which ponatinib is located. Interestingly, 54 compounds shared the pharmacophore distribution of ponatinib. Table 1 summarizes the docking scores of the compounds with the lowest binding free energies. Figure 1B shows the location of the pharmacophoric points identified for the interaction of ponatinib with the RIPK2 active site and the proposed binding modes of the ligands with the lowest binding free energies, respectively. It is noteworthy that the compounds with the lowest calculated binding free energies exhibit a high structural similarity between them [MolPort016-359-762 (labeled as RIPK2 inhibitor 1 in subsequent 
figures, 3-benzamido-4-methyl- $N$-[3-(1-methyl-1H-imidazol2yl)phenyl]benzamide), MolPort-015-752-252, MolPort-015604-588, and MolPort-016-412-727) and a high correlation with pharmacophoric points of ponatinib. These results suggest that compound MolPort-016-359-762 and its derivatives have a high probability of inhibiting RIPK2.

Structure Similarity Search. A total of 10,655,787 compounds with at least $60 \%$ similarity to ponatinib were obtained from the PubChem database to dock them into this binding site and identify new potential inhibitors. We selected the most similar ( $\geq 90 \%$; 803 compounds) and less similar $(<62 \%$; 50,000 compounds randomly selected from a total of $2,607,266)$ structures to identify ponatinib-like molecules and novel scaffolds, respectively. After filtering with Lipinski's rule and removing duplicates and inorganic molecules, we performed molecular docking studies in the RIPK2 active site using AutoDock Vina, from which more than 500 compounds with a calculated $\Delta$ Gbind $\leq-10.0 \mathrm{kcal} / \mathrm{mol}$ were identified. The docking scores of ponatinib and the compounds with the lowest binding free energies are summarized in Table 1. As might be expected, the compounds with the highest similarity in values showed similar binding modes compared with ponatinib (PC_24826801, PC_57405602, PC_58945635, PC_58945682, and PC_58945685). However, compound PC_57410628 was the only molecule with a lower binding free energy than the lead molecule. Moreover, compound PC_44716361 [MolPort-001-746-327 (labeled as RIPK2 inhibitor 2 in subsequent figures)], which presents a lower similarity to ponatinib, represents a new scaffold with unique structural features offering alternatives from a medicinal chemistry perspective. Figure 1B shows the proposed binding mode of these ligands in the RIPK2 binding site. This figure also shows that all of the compounds identified possible form hydrogen bonds between the Glu66 residue and the carboxamide group. The initial virtual screening, ranking chemical structures, and the pan-assay interference compounds check of the selected compounds (Sterling and Irwin, 2015) are reported in Supplemental Table 1.

Utilizing mass spectrometry and ${ }^{1} \mathrm{H}-\mathrm{NMR}$ (Fig. 2; Supplemental Figs. 1-3A), we confirmed $>95 \%$ purity of our two lead compounds and confirmed the structure of the identified RIPK2 inhibitors 1 and 2. ROESY spectra provided throughbond and through-space connectivities that were essential for obtaining complete chemical shift assignments of both molecules (Supplemental Figs. 1 and 2). In addition, through-space rotating-frame nuclear Overhauser enhancement (ROE) connectivities provided valuable information about the conformational preferences of RIPK2 inhibitor 1 in solution (Fig. 2B). The lack of ROEs between HN22 and either HC17 or HC25 indicates that the central methyl-containing aromatic ring is roughly perpendicular to the amide plane of HN22, as would be expected based on steric considerations. In contrast, HN13 shows ROEs to HC8, HC17, and HC21, indicating that the two central benzene rings are more planar with respect to the central amide bond of HN13, similar to the conformation seen in ponatinib (Fig. 1B). Thus, the arrangement of benzene rings found in RIPK2 inhibitor 1 resembles the geometry of the corresponding aromatic rings in ponatinib bound to RIPK2. The presence of both HN13-HC17 and HN13-HC21 ROEs suggests rotation about the C14-C16 bond, with rotomer i (Supplemental Fig. 1B) matching the conformation found in ponatinib-RIPK2.
However, the presence of an HN13-HC8 ROE coupled with the absence of an HN13-CH10 ROE suggests that rotation about the C9-N13 bond is more restricted, which may force the imidazole ring of RIPK2 inhibitor 1 into a position opposite to the corresponding $\mathrm{CF}_{3}$ group of ponatinibRIPK2 (Fig. 1B, panel iii), which would place it in a more solvent-exposed position than the buried position of the $\mathrm{CF}_{3}$ group. Further detailed analysis may be warranted to isolate these two forms and determine bioactivities for RIPK2 and $\mathrm{NF} \kappa \mathrm{B}$ inhibition.

Validation of RIPK2 Inhibitors Using Kinome Analysis. The specificity of the identified RIPK2 inhibitors to inhibit numerous kinases was explored utilizing the Kinome Scan profiling service at DiscoverX (Fremont, CA). This utilizes an in vitro kinase approach with purified recombinant kinases/peptide substrates to determine activity of kinases in the panel. We explored if our lead compounds, RIPK2 inhibitors 1 and 2, can inhibit other targets at $100 \mathrm{nM}$ in the 97 kinome panel (Supplemental Table 2A). This concentration was used due to robust inhibition of $\mathrm{NF} \kappa \mathrm{B}$ and RIPK2 kinase assay in assays outlined in Figs. 2-6. The kinome inhibition analysis revealed robust selectivity toward RIPK2 for RIPK2 inhibitor 1 with $20 \%-27 \%$ inhibition of c-ABL, Aurora kinase B, or ERBB2 (Supplemental Table 2A). RIPK2 inhibitor 2 appears to inhibit $38 \%$ of the activity of SNARK and $27 \%$ of the activity of FGFR2, GSK3$\beta$, JNK1, CSNK1G2 (casein kinase 1), and MET tyrosine kinase. These off-target inhibitions are not surprising, as it is difficult to obtain a specific small-molecule inhibitor. This would suggest possible activities beyond inhibiting RIPK2 that will need to be empirically tested. Initial empirical testing for c-ABL was carried out via an in vitro kinase approach with purified recombinant kinases at the MRC Protein Phosphorylation and Ubiquitination Unit in Dundee, Scotland. Both of our RIPK2 small-molecule lead compounds inhibited c-ABL but with an $\mathrm{IC}_{50}$ of $>100 \mu \mathrm{M}$ (Fig. 3A). Given this observation, we hope that the off-target effects on Aurora kinase B and ERBB2 (if any) will also be in a vastly different concentration range of $100 \mathrm{nM}$.

Cell-Based Confirmation of RIPK2 Inhibition and Inhibition of NF $\mathbf{B}$ Activation. We next explored cellbased in vitro inhibition of RIPK2. We can clearly observe RIPK2 inhibitor 1 and 2 inhibition of MDP-dependent activation of RIPK2 autophosphorylation (on tyrosine 474) using an in vitro kinase assay in HCT116 cells (Fig. $3 \mathrm{~B}$ ). MDP is the molecular ligand for the NOD2 pathogen receptor whereby RIPK2 can be activated. Similar results for RIPK2 inhibitor 1 inhibition of RIPK2 were obtained in breast cancer cells (BT549 and MDA-MB231 cells, data not shown). Immunodepletion using an anti-RIPK2 antibody (Fig. 3C, condition B) or Crispr/Cas9 knockout of RIPK2 (Fig. 3D) confirmed specificity of detection of the autophosphorylated RIPK2 band in Fig. 3B. Furthermore, using a phospho-tyrosine [Y] 474 (in-house generated) and a phospho-serine [S] 176 (Cell Signaling, Inc., Danvers, MA) RIPK2 antibodies, we confirmed that active RIPK2 is recognized by these antibodies upon activation with MDP treatment in 293HEK fibroblast and HCT116 colon cancer epithelial cells (Fig. 3E, left panel) and detection of constitutively active RIPK2 in the colon cancer cell line SW480 (Fig. 3E, right panel). Interestingly, we can observe constitutively active RIPK2 in Hodgkin's lymphoma cell lines HDMYZ, L428 (Fig. 4A), and KMH2 cells (Fig. 4B). This signal 


\section{A Mass Spectrometry Confirmation of RIPK2 Inhibitor 1 Formula Weight}

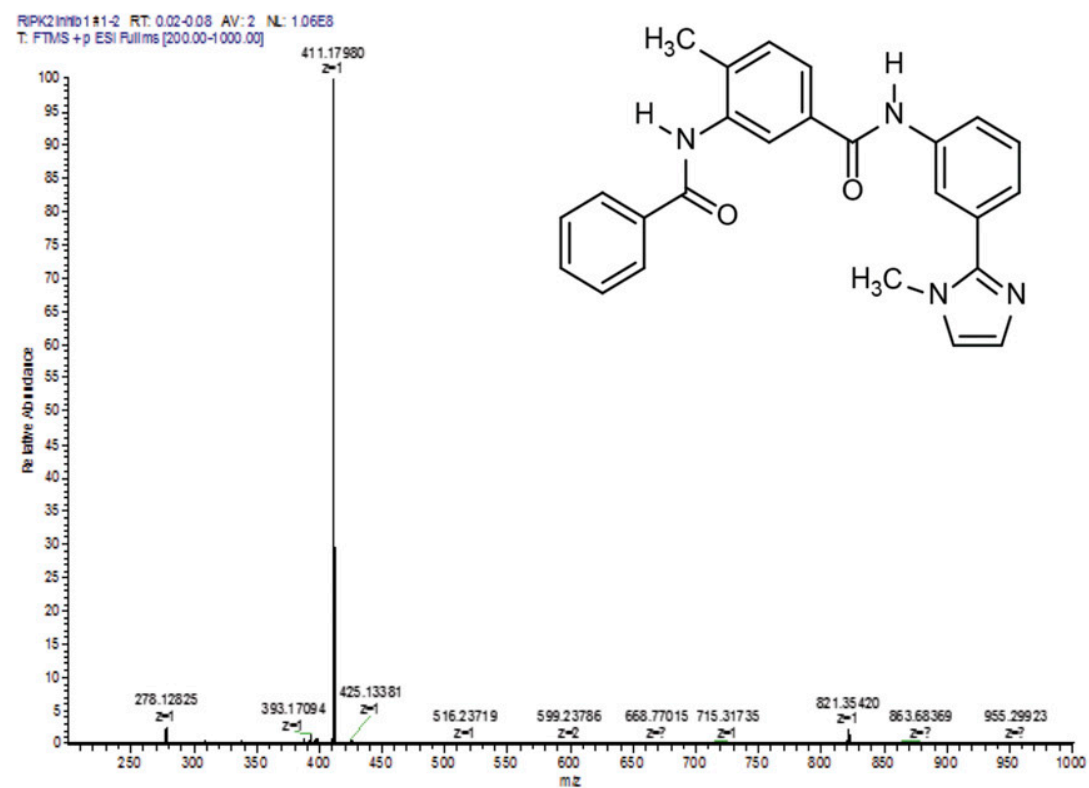

B

RIPK2 Inhibitor 1 - Two Dimensional 1H-1H ROESY Spectrum

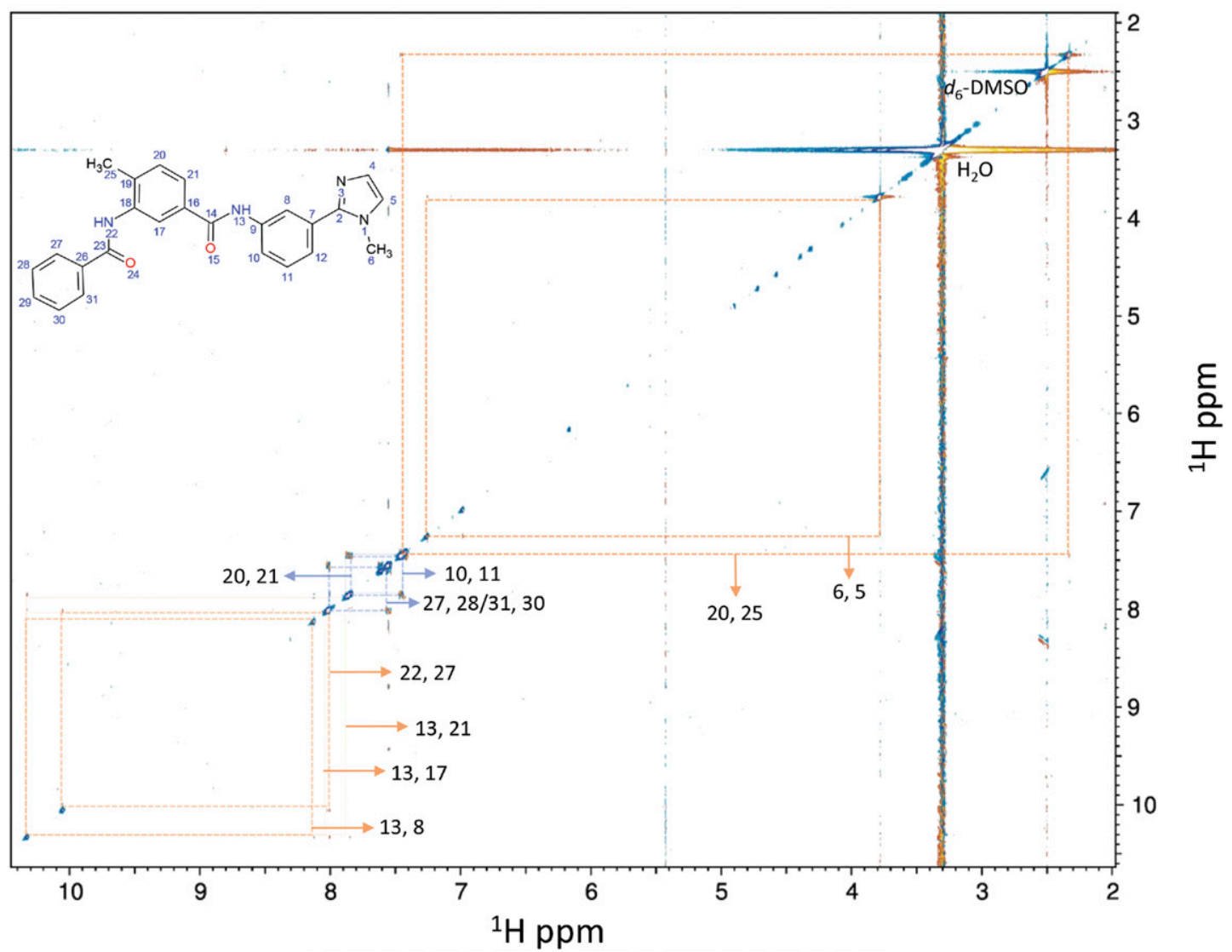

Fig. 2. Mass spectrometry and NMR confirmation of mass and structure of RIPK2 Inhibitor 1. (A) Direct-infusion measurements were carried out on an LTQ Orbitrap XL (Thermo Scientific) mass spectrometer using the Ion Max ESI source. Mass confirmation was obtained as indicated. (B) Twodimensional ${ }^{1} \mathrm{H}-{ }^{1} \mathrm{H}$ ROESY spectrum of 3-benzamido-4-methyl- $N$-[3-(1-methyl-1H-imidazol-2-yl)phenyl]benzamide. Boxes (orange) represent throughspace connectivities between protons in the compound. Blue boxes are signals due to three-bond $J$-coupling between aromatic ring protons. Four-bond $J$-coupling signal is less intense. AV, number of scans that were averaged; FTMS, Fourier Transform Mass Spectrometry; NL, normalized level; RT, retention time. 
A

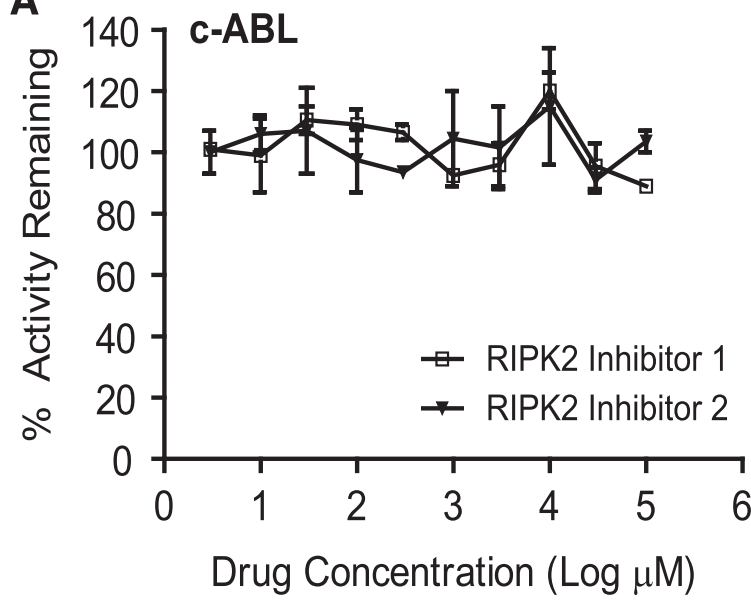

C Immunodepletion of RIPK2 results in loss of RIPK2 IVK Signal
B RIPK2 Inhibitor modulation
of RIPK2 autophosphorylation HCT116 Cells

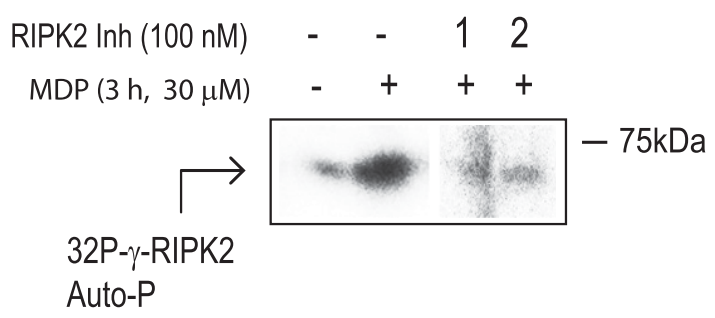

D

\section{Crispr/Cas9 knockdown of RIPK2 negates RIPK2 autophosphorylation}

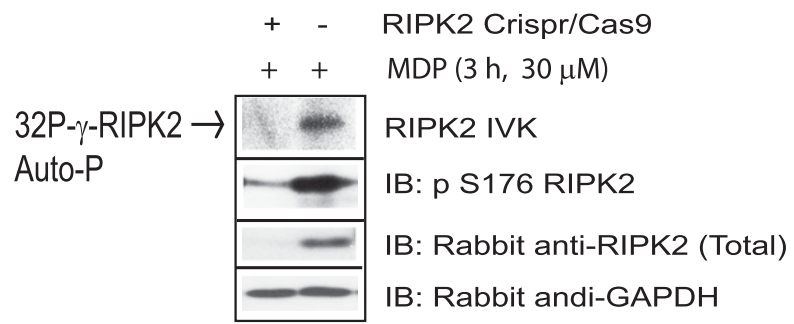

HCT116 Cells
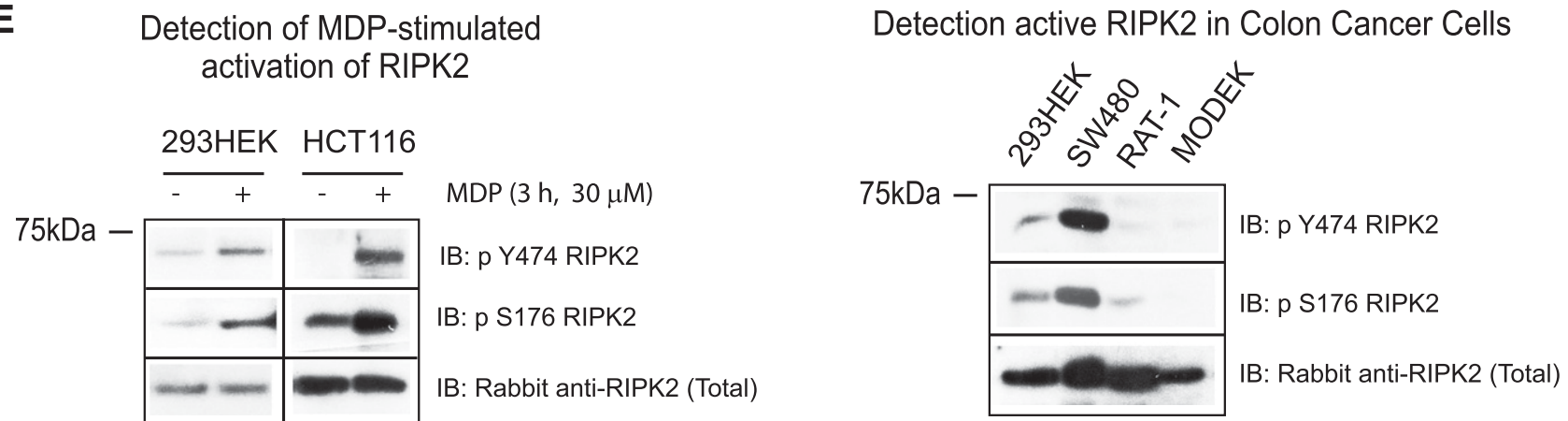

Fig. 3. Analysis of RIPK2 kinase activity. (A) Kinase activity for c-ABL was tested in the presence of inhibitors 1 and 2 . $\mathrm{IC}_{50}$ for c-ABL for both inhibitors is $>100 \mu \mathrm{M}$. Analysis was carried out using purified c-ABL and the substrate peptide, EAIYAAPFAKKK, in association with the MRC Unit on Phosphorylation and Ubiquitination, University of Dundee, Scotland. (B) RIPK2 autophosphorylation (Auto-P) at site Y474 is shown. Inhibition was carried out on cells followed by lysis and IP with rabbit anti-RIPK2 overnight. Following protein G pull down and wash, ${ }^{32} \mathrm{P}-\gamma$-ATP was added and kinase reaction was allowed to proceed for 30 minutes at $30^{\circ} \mathrm{C}$. Following kinase reaction, SDS-PAGE was used to separate out the proteins, and gel was then dried and exposed to film. (C) RIPK2 in HCT116 cells were IP with an anti-RIPK2 antibody (A) and the supernatant after IP in (A) was IP in (B) with the same RIPK2 antibody, and in vitro kinase (IVK) assay was carried out on both samples. (D) Crispr/Cas9 knockout of RIPK2 in HCT116 colon cells was carried out, followed by MDP activation and RIPK2 IVK analysis to illustrate use of RIPK2 Crispr/Cas9 reagents and specificity of our IVK assay. (E) Left panel: cells were stimulated with MDP for 3 hours, and RIPK2 activation was monitored by a phosphotyrosine (pY) 474 RIPK2 antibody (in-house antibody) and phosphoserine (pS)176 phosphorylation (Cell Signaling). Right panel: several colon cancer cells were investigated for constitutive activation of RIPK2 using phosphorylation specific antibodies to pY474 and pS176 as indicated. Bottom: constitutive activation of RIPK2 in numerous cell lines are explored. GAPDH, glyceraldehyde-3-phosphate dehydrogenase; IB, immunoblotting. 


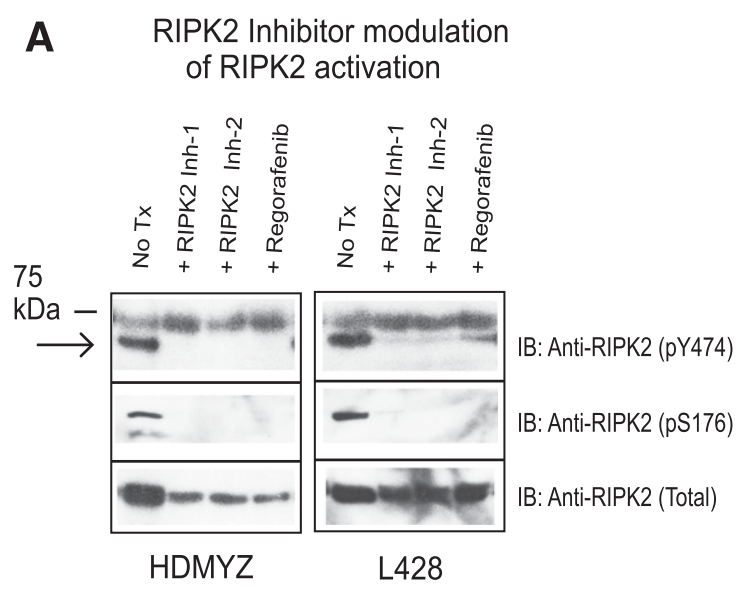

$\mathbf{B}$

\section{RIPK2 Inhibitor 1 Modulation of RIPK2 Autophosphorylation}
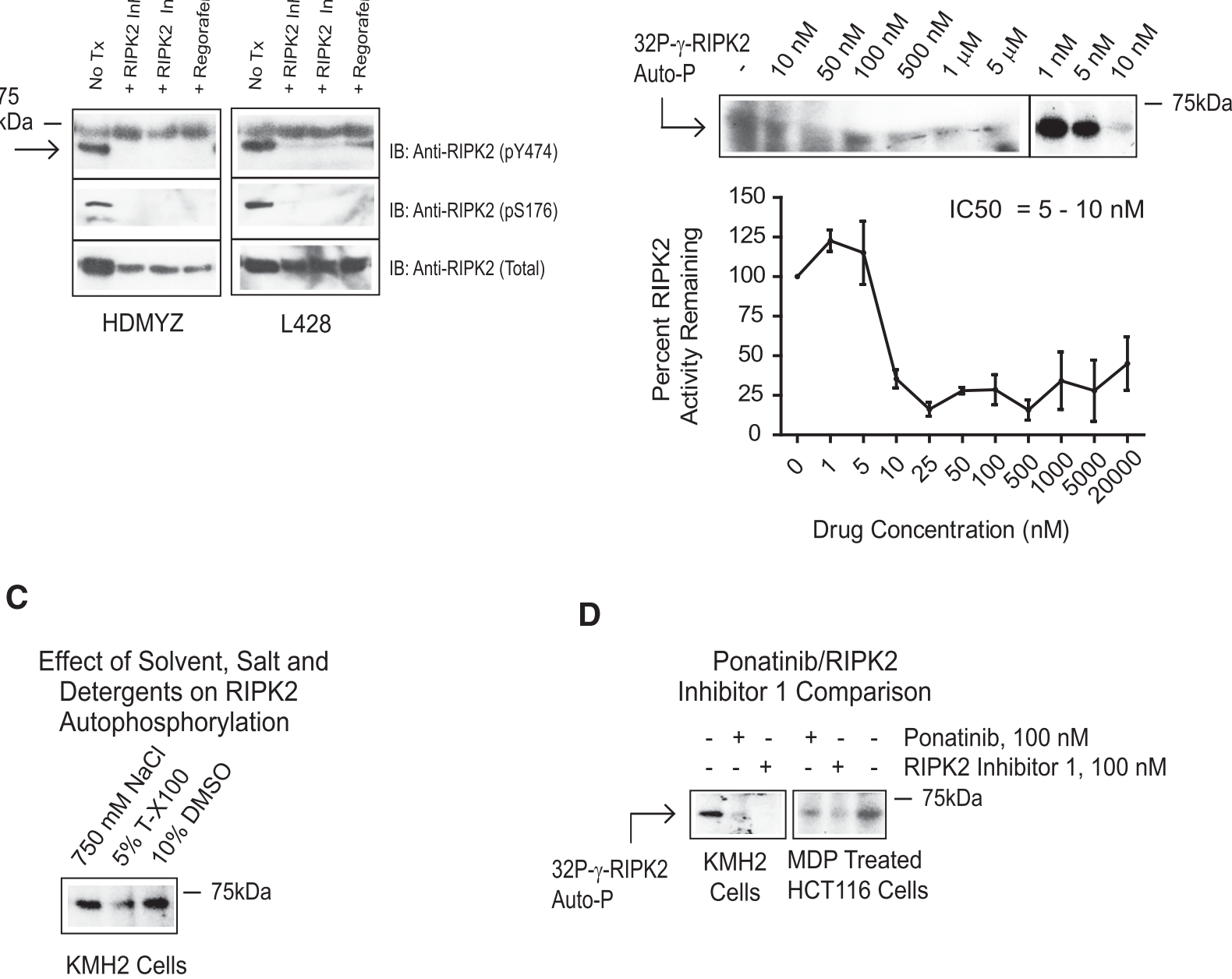

D

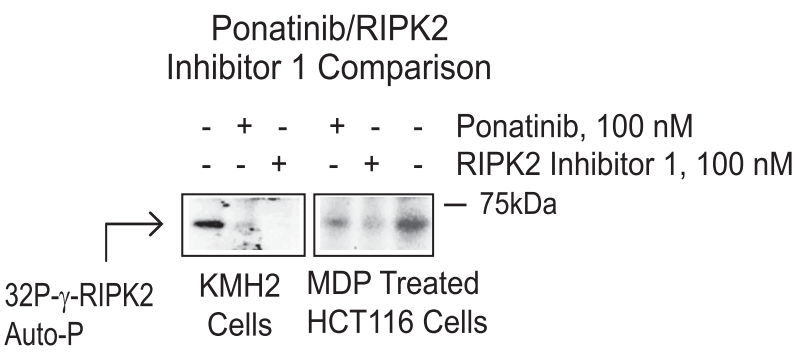

Fig. 4. Characterization of RIPK2 kinase inhibitors. (A) Immunoblot illustration of the use of RIPK2 phospho-antibodies in two Hodgkin's lymphoma cells that have constitutive active RIPK2. A comparison with a known RIPK2 inhibitor is shown (regorafenib). All inhibitor concentrations were $100 \mathrm{nM}$. Similar results were observed for a third Hodgkin's lymphoma cell line, KMH2 [see (B)]. (B) KMH2 Hodgkin's lymphoma cells were inhibited in vivo for 33-36 hours using the indicated concentration of RIPK2 inhibitor 1. (Top) An in vitro kinase assay was carried out by IP overnight with $1 \mu \mathrm{g}$ of the rabbit anti-RIPK2 antibody from ProteinTech (Rosemont, IL) and $1 \mathrm{ml}$ of lysate from a confluent six-well dish of KMH2 cells. Immune complexes were separated by SDS-PAGE and captured by autoradiography. (Bottom) Quantitation of three to five independent experiments to reveal an approximate $\mathrm{IC}_{50}$ of 5-10 $\mathrm{nM}$ for in vivo RIPK2 inhibition. (C) Analysis of effect of drug solvent (DMSO), salt concentration, and detergents on the kinase activity of RIPK2. Following IP, the indicated reagents were added for 10 minutes followed by $32-\gamma$-ATP to initiate the kinase reaction as described in the Materials and Methods section. (D) RIPK2 in vitro kinase assay was carried out as in Fig. 3B in KMH2 and HCT116 cells in the presence of ponatinib or RIPK2 inhibitor 1 to carry out a comparison of RIPK2 inhibition. IB, immunoblotting.

can effectively be inhibited using RIPK2 inhibitors 1 and 2 and regorafenib (a known RIPK2 kinase inhibitor) at $100 \mathrm{nM}$ as determined using RIPK2 phospho-specific antibodies (Fig. 4, $\mathrm{A}$ and B). Furthermore, we determined that RIPK2 inhibitor 1 effectively inhibited KHM2 cells at an approximate $\mathrm{IC}_{50}$ of 5-10 nM, and kinase activity was somewhat sensitive to detergent conditions (Fig. 4, B and C). When compared with inhibition with ponatinib (a known RIPK2 inhibitor and chemical template for selecting our RIPK2 small molecule), our RIPK2 inhibitor performed equally to inhibit RIPK2 kinase activity in an in vitro kinase assay in two different cell lines (Fig. 4D).

The functional consequence of inhibiting MDP-dependent activation of RIPK2 is the loss of $\mathrm{NF}_{\kappa} \mathrm{B}$ activity (Fig. 5; Supplemental Fig. 3B) and DNA binding ability, especially for the interleukin-8 (IL-8) promoter (Supplemental Fig. 4A). Not surprisingly, RIPK2 inhibitor 1 was more efficacious at $100 \mathrm{nM}$ at inhibiting $\mathrm{NF}_{\kappa} \mathrm{B}$ activity when compared with RIPK2 inhibitor 2 , gefitinib, or regorafenib ( $P$ values comparing RIPK2 inhibitor $1 \mathrm{vs.} \mathrm{RIPK2} \mathrm{inhibitor} \mathrm{2,} \mathrm{gefitinib,} \mathrm{and}$ regorafenib inhibition of MDP-driven $\mathrm{NF}_{\kappa} \mathrm{B}$ activation were $0.0015,0.0006$, and 0.004 , respectively). However, RIPK2 inhibitor 1 was as efficacious as ponatinib at $100 \mathrm{nM}$ for $\mathrm{NF}_{\kappa} \mathrm{B}$ inhibition $(P$ value $=0.06$ when comparing RIPK2 inhibitor 1 vs. ponatinib inhibition of MDP-driven $\mathrm{NF}_{\kappa} \mathrm{B}$ activation). Interestingly, a recently characterized RIPK2 inhibitor, GSK-583 (Haile et al., 2016), did not inhibit MDPdependent $\mathrm{NF}_{\kappa} \mathrm{B}$ activity at $100 \mathrm{nM}$ in HCT116 cells (data not shown), although it is known to in primary immune cells. Interestingly, we did observe a small but significant 


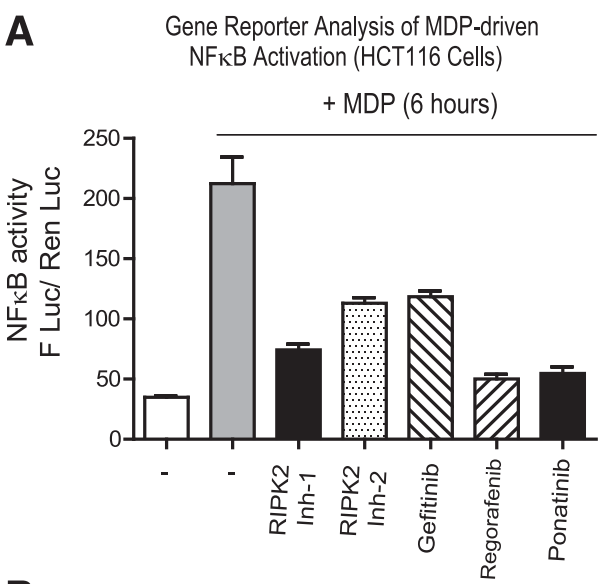

B
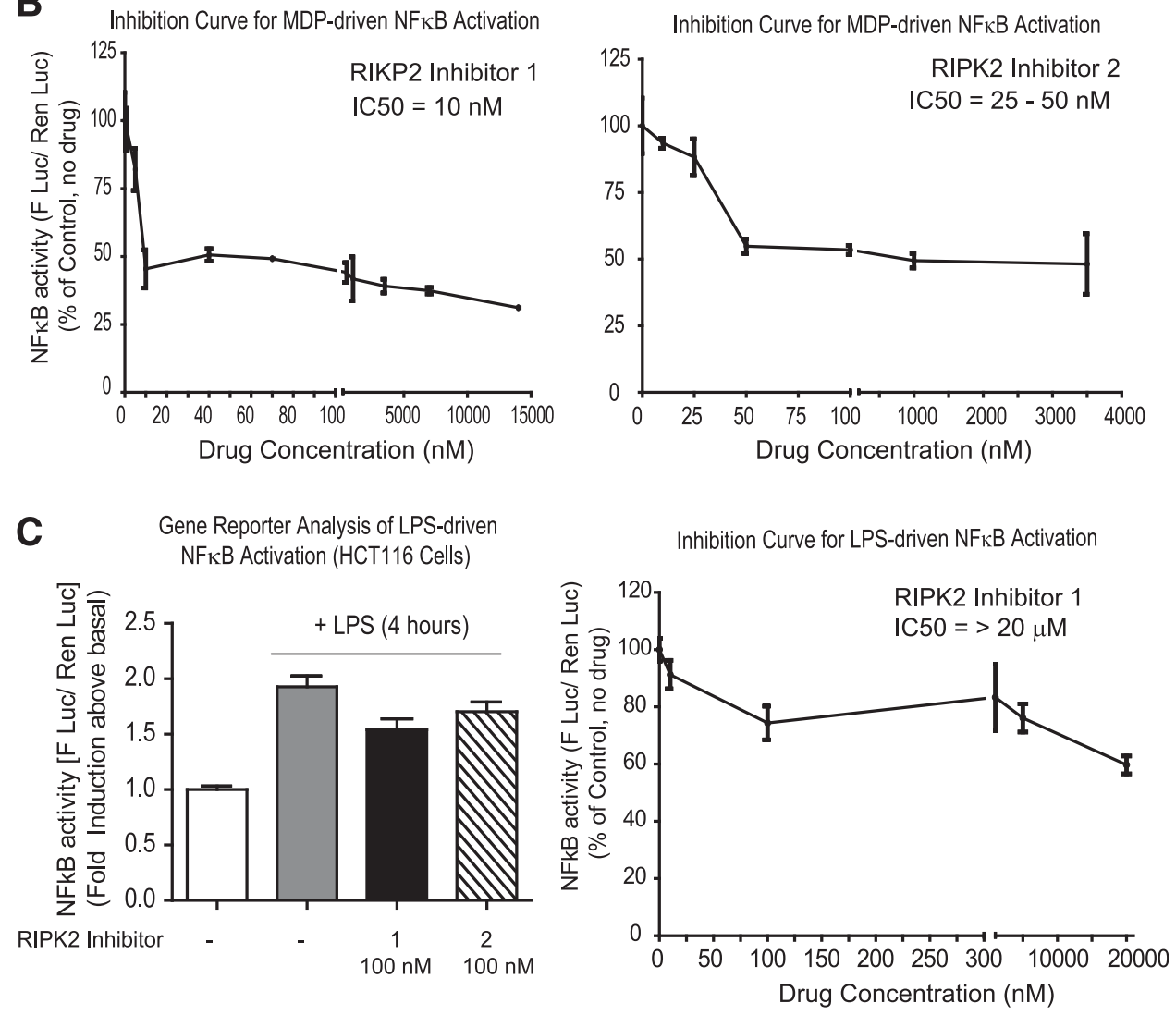

Fig. 5. RIPK2 Inhibition of $\mathrm{NF}_{\kappa} \mathrm{B}$ activity. (A and $\mathrm{B}$ ) $\mathrm{NF}_{\kappa} \mathrm{B}$ gene reporter assay determination of inhibition of MDPstimulated $\mathrm{NF}_{\kappa} \mathrm{B}$ activity with RIPK2 inhibitors (A) and an approximate $\mathrm{IC}_{50}$ determination for inhibition of MDP driven- $\mathrm{NF}_{\kappa} \mathrm{B}$ activation using RIPK2 inhibitors 1 and 2 (B). All drugs in (A) were used at $100 \mathrm{nM}$. $P$ values for RIPK inhibitor treated vs. MDP (no drug) were $<0.0001$ (inhibitor 1), 0.06 (inhibitor 2), <0.006 (gefitinib), 0.0002 (regorafenib), and 0.0008 (ponatinib). For ponatinib, $n=4$ and $n=10$ for the rest with approximate $\mathrm{IC}_{50}$ curves shown in (B). (C) Left panel: $\mathrm{NF}_{\kappa} \mathrm{B}$ gene reporter assay determination of inhibition of LPS-stimulated $\mathrm{NF}_{\kappa} \mathrm{B}$ activity with RIPK2 inhibitors (concentration as indicated). $P$ values for RIPK2 inhibitor treated vs. LPS (no drug) were $<0.02$ (inhibitor 1 ) and $<0.268$ (for inhibitor 2) $(n=6)$. Right panel: approximate $\mathrm{IC}_{50}$ determination for inhibition of LPS-NF $\kappa$ B-driven inflammation using RIPK2 inhibitors 1 and 2 (B). reduction in lipopolysaccharide (LPS; via TLR4 and TLR2), $\mathrm{TNF} \alpha$, and IL-1 $\beta$-dependent activation of $\mathrm{NF} \kappa \mathrm{B}$ (Fig. $5 \mathrm{C}$ and an approximate $\mathrm{IC}_{50}$ in Fig. 5C, right panel; Supplemental Fig. 3B). Although a reduction, the approximate $\mathrm{IC}_{50}$ for RIPK2 inhibition of LPS-driven $\mathrm{NF}_{\kappa} \mathrm{B}$ activation is $>20 \mu \mathrm{M}$, and we speculate it will also be much higher for inhibition of $\mathrm{TNF} \alpha$ and IL-1 $\beta$-dependent activation of $\mathrm{NF}_{\kappa} \mathrm{B}$. Interestingly, RIPK2 inhibitor 1 can also inhibit the activation of hypoxia-response element I response to chemical induction using $1 \% \mathrm{H}_{2} \mathrm{O}_{2}$ (Supplemental Fig. 3C) to suggest either a link to inflammation or a direct modulation of hypoxiainducible factor $\alpha$ function. These observations validate the specificity of our RIPK2 inhibitor for NOD/RIPK2 biology and usefulness as an in vivo inhibitor of MDP-driven inflammation.
RIPK2 Inhibitors Can Inhibit the Proliferation of Several Cancer Cells but Do Not Promote Apoptosis or Cell Cycle Arrest. Inflammation is a strong driver of malignant transformation and abnormal proliferation, especially in the colon (Lasry et al., 2016) and breast (Suman et al., 2016). We explored the ability of RIPK2 inhibitors to reduce the proliferative rate of highly metastatic cancer cells. For most of the cancer cells, 45-100 nM RIPK2 inhibitor 1 inhibited $>70 \%$ of the proliferation of colon and blood cancer cells while preserving the proliferation of normal Rat-1 and ModeK intestinal epithelial cells (Fig. 6A). These data would suggest strong suppressive properties of RIPK2 inhibitor 1 and, to some extent, inhibitor 2 on cell proliferation. Regorafenib, a recently characterized RIPK2 inhibitor, did not significantly inhibit the proliferation of these cells in an 
A

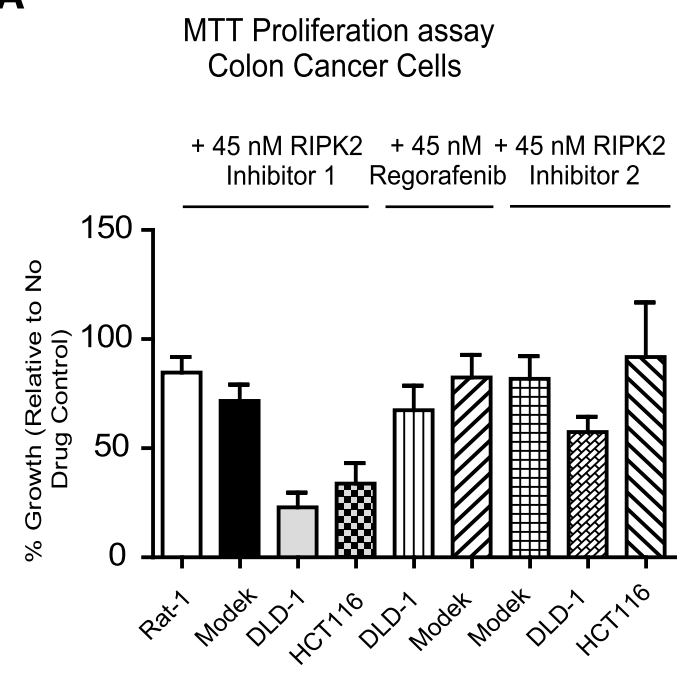

C

In vivo inhibition of lung inflammation inury
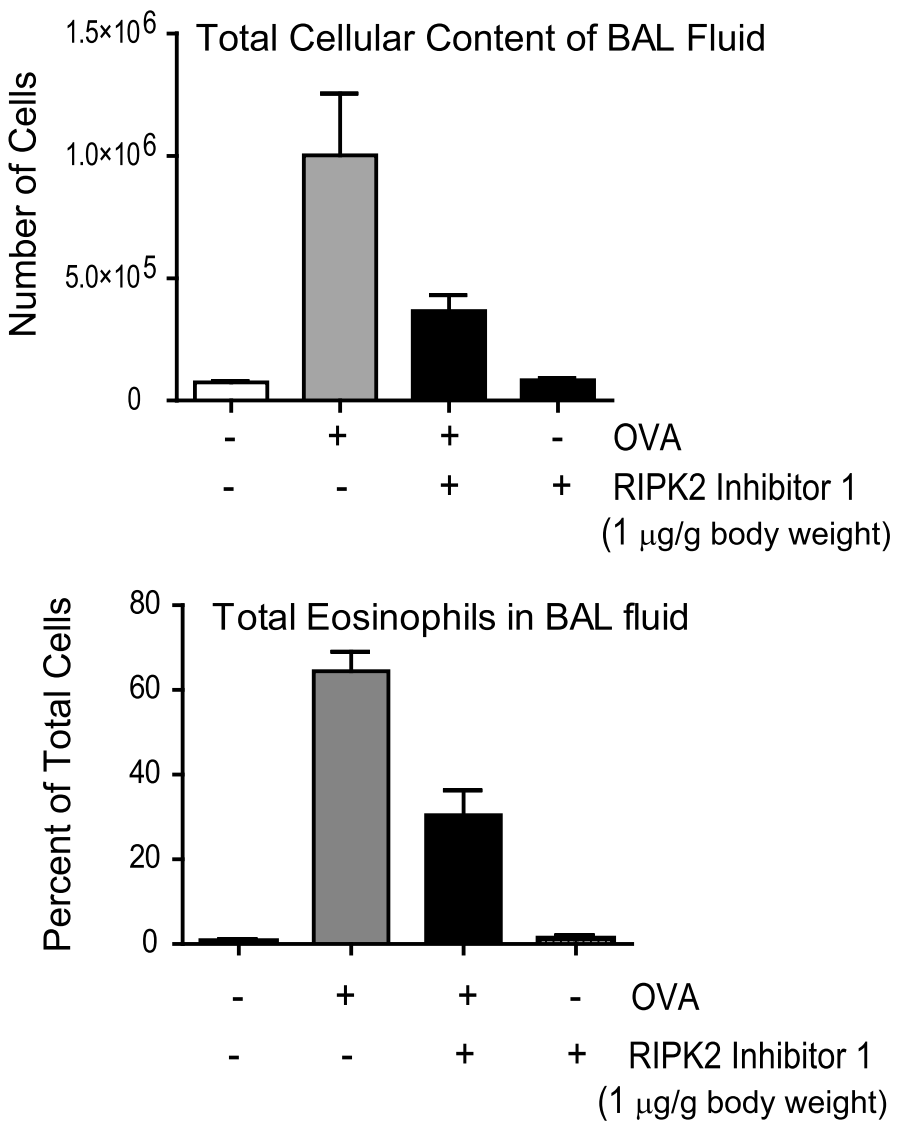

In vivo inhibition of intestinal inflammation injury

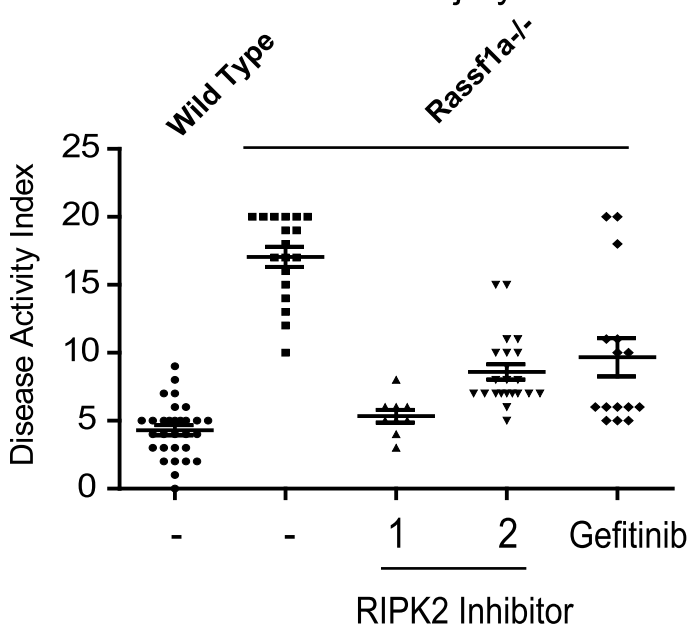

D

Fig. 6. In vivo inhibition of proliferation, intestinal, and lung inflammation using RIPK2 inhibitors. (A) RIPK2 inhibitors modulate the proliferation of several colon cancers but not normal cells. MTT assay was carried out with the indicated concentrations of RIPK2 inhibitors in the indicated cell lines. HCT116 and DLD-1 are colon cancer cell lines, whereas RAT-1 and MODEK are normal rat and mouse intestinal cell lines, respectively. RIPK2 inhibitor 1 does not appear to interfere with proliferation of normal intestinal cells (Rat- 1 and ModeK) at $45 \mathrm{nM}$. For all experiments, $n=5-29$. $P$ value of HCT116 (+ inhibitor 1) vs. HCT116 (+ inhibitor 2) was <0.03; DLD-1 (+ inhibitor 1) vs. DLD-1 (+ inhibitor 2) was $<0.02$; DLD-1 (+ inhibitor 1) vs. DLD-1 (+ regorafenib) was $<0.006$. For RIPK2 inhibitor 1-treated DLD-1 or HCT116 vs. RIPK2 inhibitor 1-treated ModeK or Rat-1 cells (normal cells), $P<0.002$ (analysis in either cell type); for RIPK2 inhibitor 2 -treated DLD-1 or HCT116 vs. RIPK2 inhibitor 2-treated ModeK cells (normal cells), $P<0.02$ and 0.098 (analysis in DLD-1 or HCT116 cells, respectively); for regorafenib-treated DLD-1 vs. regorafenib-treated ModeK (normal cells), $P<0.2$ but $>0.05$. (B) Intestinal inflammation injury was carried out using the DSS model. DSS is taken up in the drinking water and migrates to the colon to cause irritation and localized inflammation. Rassf1 $1 a^{-1-}$ mice are extremely sensitive to this model, with $<25 \%$ survival following a $3 \%$ DSS insult for 7 days followed by water for 7 days. Most Rassf1a ${ }^{-1-}$ mice require euthanasia by days 7-9. Disease activity is scored based on rectal bleeding, piloerection, movement, and 
TABLE 2

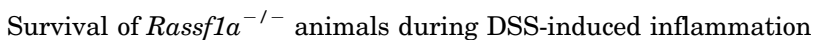

The presence of RIPK2 inhibitors reduced acute intestinal inflammation and enhanced overall survival of the mice.

\begin{tabular}{llr}
\hline \multicolumn{1}{c}{ RIPK2 Inhibitor } & Treatment and Genotype & Percentage Survival \\
\hline No drug & DSS-treated Rassf1a ${ }^{-/-}$ & $7 / 30=22 \%$ \\
1 (MolPort-016-359-762) & DSS-treated Rassf1a ${ }^{-\prime}$ & $8 / 11=73 \%$ \\
2 (MolPort-001-746-327) & DSS-treated Rassf1a ${ }^{-/}$ & $5 / 9=56 \%$ \\
Gefitinib & DSS-treated Rassf1a & $7 / 15=47 \%$ \\
\hline
\end{tabular}

MTT assay (3-(4,5-dimethylthiazol-2-Yl)-2,5-diphenyltetrazolium bromide) (Fig. 6A). We also observed inhibition of triple negative breast cancer cells, HCT1143 and MDA-MB231, with RIPK2 inhibitor 1 (Supplemental Fig. 4B), suggesting effective antiproliferative properties. The approximate $\mathrm{IC}_{50}$ for inhibition was around $30-60 \mathrm{nM}$ for most of the cells tested (two are shown in Supplemental Fig. 4C). This effect on growth inhibition was not due to alterations in cell cycle (Supplemental Fig. 5) nor a significant increase in the apoptotic cell populations, suggesting that RIPK2 inhibition of proliferation is independent of cell cycle or apoptotic changes.

RIPK2 Inhibitors Do Not Inhibit RIP1-Directed Cell Death, Ferroptosis, or Modulation of Mitochondrial Physiology. The RIPK family of proteins are actively involved in numerous cell-death processes beyond death receptor-dependent cell death (Vanden Berghe et al., 2016). We characterized two forms of cell death involving erastinstimulated ferroptosis (an iron-dependent form of cell death, Supplemental Fig. 6A) and VAD-fmk, Smac Mimetic-164, and $\mathrm{TNF} \alpha$ stimulation of RIPK1-directed cell death (Supplemental Fig. 6B). In both cases, RIPK2 inhibitor 1 did not interfere with these forms of cell death to support our cell-cycle effects and to suggest no overlap with RIPK1 biology (Supplemental Fig. 6A). Recently, RIPK3 was demonstrated to require a Bax/Bak effect on the mitochondrial permeability transition pore to carry out necroptosis, and the importance of both RIPK1 and RIPK3 in relation to necroptosis-directed degenerative, inflammatory, and infectious diseases has been published (Moriwaki et al., 2015). We thus explored how the identified RIPK2 inhibitors can perturb mitochondrial physiology by evaluating the activity of the sirtuins, a class of deacetylases that use $\mathrm{NAD}^{+}$to remove acetyl groups from proteins (Tang, 2016). Indeed, the putative SIRT1 activator resveratrol can stimulate mitochondrial biogenesis to promote increased wellness in the individual (Ostojic, 2017). Therefore, we explored the effect on sirtuin activity using the BIOMOL assay (Enzo Life Sciences, Farmingdale, NY) as described previously (Dai et al., 2016). We observed no statistically significant increase in SIRT1 activation when using the compounds at 0.1 or $10 \mu \mathrm{M}$, indicating that phenotypes observed for the use of RIPK2 inhibitors at these doses in cells are likely through SIRT1-independent mechanisms when compared with the effect of resveratrol (Supplemental Fig. 6C). The identified RIPK2 inhibitors are thus selective inhibitors of RIPK2-dependent $\mathrm{NF}_{\kappa} \mathrm{B}$ pathways if we consider the data in Fig. 5 and Supplemental Figs. 3B, 4, and 5.

RIPK2 Inhibitors Can Efficiently Resolve Intestinal Inflammation in an Ulcerative Colitis Model. DSSinduced intestinal inflammation is a model for ulcerative colitis [a form of inflammatory bowel disease (IBD)]. DSS functions to irritate the colonic mucosa to promote localized inflammation, active cell death, and localized destruction of the epithelial barrier to the lumen of the colon (Dieleman et al., 1998). These events subsequently drive inflammation damage indicative of what IBD patients encounter. NOD2 mutations have been observed in IBD patients, lending support for dysregulated NOD2/RIPK2 signaling driving inflammation in IBD patients (Branquinho et al., 2016). We have demonstrated the importance of Ras association domain family protein 1A [RASSF1A (or 1A)] in the pathogenesis of colitis in a rodent model (Gordon et al., 2013). RASSF1A is a tumor suppressor involved in TNF-R1-dependent apoptosis, cell cycle control, and restriction of $\mathrm{NF}_{\kappa} \mathrm{B}$ activation (Gordon et al., 2013). The Rassf1a knockout mice (both heterozygous and homozygous forms) are very susceptible to DSS-induced inflammation injury (Gordon et al., 2013) mainly due to uncontrolled inflammation linked to the NOD2/RIPK2 pathway (Said et al., unpublished observation).

Therefore, we carried out intraperitoneal injection of $1 \mu \mathrm{g} / \mathrm{g}$ body weight of RIPK2 inhibitor 1 or 2 on days 5,7 , and 9 to offset the preinflammation damage stage, peak inflammation damage stage, and postinflammation damage/restitution phase. Using this treatment scheme, we observed a robust difference on day 9 in the disease activity indices of wild-type and Rassf1a knockout mice that was significantly inhibited with the use of the RIPK2 inhibitors, especially RIPK2 inhibitor 1 (Fig. 6B; Table 2). Interestingly, a newly characterized RIPK2 inhibitor, gefitinib, can also inhibit intestinal inflammation injury but only promote a $41 \%$ survival versus $73 \%$ survival with RIPK2 inhibitor 1 (Fig. 6B; Table 2). Similar results were obtained for the protein tyrosine kinase inhibitor regorafenib (data not shown). Since both gefitinib and our RIPK2 inhibitors can inhibit RIPK2, either our drug

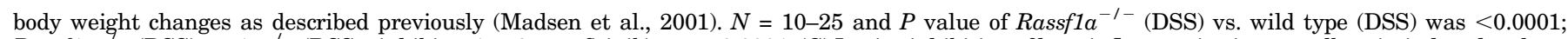

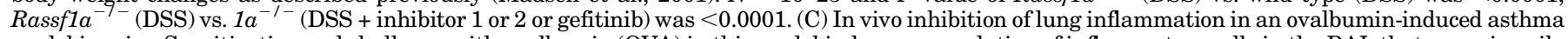

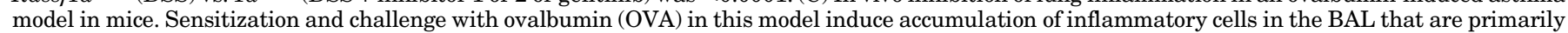

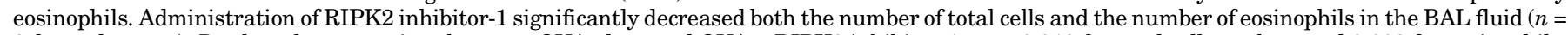

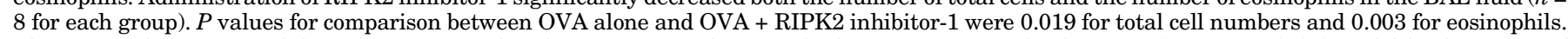

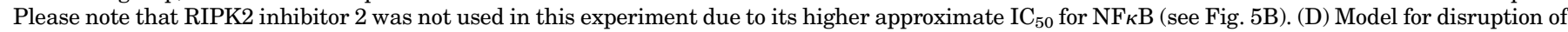

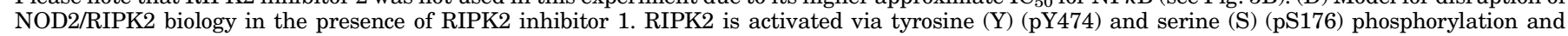

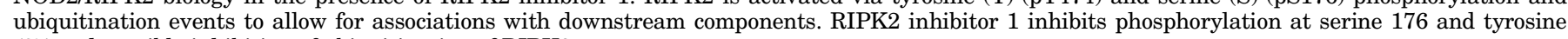
474 and possible inhibition of ubiquitination of RIPK2. 
has more affinity for RIPK2 or the off-target effects of RIPK2 inhibitor 1 are beneficial to aid in recovery from inflammation injury using the DSS model.

We then explored pharmacokinetic properties of RIPK2 inhibitor 1 in the sera of mice. Analysis revealed that RIPK2 Inhibitor 1 can be eluted at $\sim 3.7$ minutes in a region of the chromatogram free of interfering substances (Supplemental Fig. 6D, top panel). After a dose of $1-2 \mathrm{mg} / \mathrm{kg}$ IP of inhibitor, the compound was not quantifiable in mouse serum. However, after dosing mice with $15 \mathrm{mg} / \mathrm{kg}$ IP, the RIPK2 inhibitor was of measurable concentrations in most of the serum samples assayed. The mean $\mathrm{C}_{\max }$ was $114 \mathrm{ng} / \mathrm{ml}$ occurring at 1 hour after dosing, and the area under the serum concentration versus time curve was $573 \mathrm{ng} \times \mathrm{h} / \mathrm{ml}$, with a terminal phase half-life of 1.9 hours observed. Therefore, it appears that RIPK2 inhibitor 1 may be efficiently metabolized within 2 hours and cleared from the blood (Supplemental Fig. 6D, bottom panel). Furthermore, toxicity analysis carried out 9 days after the last dose of intraperitoneal injection of $2 \mu \mathrm{g} / \mathrm{g}$ body weight revealed little or no changes in $>98 \%$ of the markers characterized in a complete blood count (Supplemental Fig. 7). However, creatine kinase activity was significantly elevated in animals treated with RIPK2 inhibitor 1 as opposed to RIPK2 inhibitor 2. During treatment with RIPK2 inhibitor 1 (and post-treatment), we did not overtly observe phenotypic changes in these animals nor notice evidence of health conditions to support a creatine kinase abnormality (such as skeletal muscle defect and movement abnormalities or unexpected euthanasia due to a cardiac abnormality). Interestingly, it has been shown that the creatine/phosphocreatine pathway may play a central role in energy metabolism, and nutritional creatine supplementation has been shown to impart beneficial effects in a number of diverse disease pathologies (Kitzenberg et al., 2016). We speculate this to be the case, as resetting of metabolic abnormalities is needed for recovery of IBD patients who have metabolic syndrome disorder (Goncalves et al., 2015). Further analysis is required to determine if elevated creatine kinase affects the health of the animal, how sustained it is, and if it can be alleviated with RIPK2 inhibitor 1 analogs. In addition, detailed analysis is needed on how our RIPK2 inhibitor 1 is metabolized and removed from the blood, where it accumulates, or if it can cross the blood-brain barrier. These are all unanswered questions that we are currently addressing.

RIPK2 Inhibitors Can Also Efficiently Resolve Lung Inflammation in an Asthma Model. RIPK2 has also been implicated in allergic airway inflammation. RIPK2 gene silencing in the airways decreased allergic airway inflammation in an ovalbumin-mediated mouse model of asthma (Jun et al., 2013). Furthermore, an association between a RIPK2 promoter polymorphism and childhood severe asthma has been shown in a Japanese population (Nakashima et al., 2006). Since we observed robust inhibition of $\mathrm{NF}_{\kappa} \mathrm{B}$ in the presence of RIPK2 inhibitor 1 and a $>70 \%$ recovery of animals from DSS-induced inflammation injury (Figs. 4 and 6B), we proceeded to explore the ability to resolve lung inflammation using the ovalbumin challenge model (Kumar et al., 2008). Ovalbumin elicits a robust inflammatory reaction in the airways characterized by an increased number of total cells and eosinophils in the BAL of ovalbumin-sensitized mice (Fig. $6 \mathrm{~B}$ ). In the presence of RIPK2 inhibitor 1 (and, to some extent, with RIPK2 inhibitor 2), we observed a significant reduction in both total cell numbers and eosinophil numbers in BAL fluid. These data suggest effective inhibition of lung inflammation.

\section{Discussion}

RIPK2 is the obligate kinase to the NOD2 pathogen receptor pathway and has been demonstrated to be involved in $\mathrm{NF}_{\kappa} \mathrm{B}$ activation and metastatic behavior in some cancers (Jun et al., 2013) and has a distinct activity versus RIPK1, 3, or 4 (Chirieleison et al., 2016). In addition, several reports suggest involvement in the activation of the immune response upon viral infection (Lupfer et al., 2013) and the requirement for the NOD2/RIPK2 molecular pathway in several models on inflammatory diseases, including experimental colitis (Branquinho et al., 2016) and arthritis (Vieira et al., 2012). Colorectal cancer (CRC) is only one example of a disease that can arise from a prior state of persistent or chronic inflammation. Individuals with IBD and primary sclerosing cholangitis (inflammation of the bile ducts) are at a much higher risk for progressing to CRC and require closer management of their debilitating disease (Dyson and Rutter, 2012; Williamson and Chapman, 2015). As such, therapies to modulate and control inflammation are robust cancer-prevention strategies.

Herein, we performed pharmacophore and similarity-based search and molecular docking studies toward the identification of potential novel RIPK2 inhibitors. In the pharmacophore search, we identified several molecules that share the pharmacophore arrangement with ponatinib. From these, compound MolPort-016-359-762 is one of the most attractive molecules to be tested since it exhibits a high correlation with the pharmacophore model and lowest binding free energy (highest score). Moreover, the similarity-based virtual screening predicted 10 molecules as new potential RIPK2 inhibitors. The lower docking score of one of the less-similar compounds, MolPort-001-746-327, presents a more attractive scaffold to be repositioned as an inhibitor of this enzyme. Interestingly, several analogs of MolPort-016-359-762 also showed higher affinities for the active site pocket of RIPK2, suggesting a high preference of this scaffold for the active site pocket. These analogs may even afford a greater selectivity to inhibit RIPK2 (data not shown). We are currently exploring the use of these analogs of MolPort-016-359-762.

As mentioned earlier, several RIPK2 inhibitors have been characterized. However, like most RIPK2 inhibitors, at $100 \mathrm{nM}$, OD36 and OD38 can also inhibit 10-FYN protoocogene, transforming growth factor 2 beta, activin receptor like kinase 2 , and Lck by $>80 \%$. Our identified RIPK2 inhibitors reveal lower binding free energies that other RIPK2 inhibitors identified, suggesting that our inhibitor may be more active. Furthermore, our identified RIPK2 inhibitors appear to have more affinity for the active site of RIPK2 than others, are more efficacious at inhibition of proliferation, and can effectively resolve lung inflammation and intestinal inflammation more robustly than gefitinib (Fig. 6B; Table 2). The efficacious quality of RIPK2 inhibitor 1 in resolving intestinal inflammation may arise from the fact that it can have a small but significant effect on TNF $\alpha$-, IL-1 $\beta$-, and LPSdriven $\mathrm{NF}_{\kappa} \mathrm{B}$ activation as seen in Fig. 5 and Supplemental Fig. 3B. Most inflammatory diseases are complex diseases that have a multitude of inflammatory pathways targeting the area. Thus, to effectively resolve inflammation in these areas, 
a broad-spectrum inhibitor may be needed to target multiple TLRs and pathogen receptors (Murgueitio et al., 2017).

All of our in vivo inhibition was at $1-2 \mu \mathrm{g} / \mathrm{g}$ body weight, which is much lower than the level used for most smallmolecule inhibitors. Preliminary pharmacokinetics suggest a 1.89-hour half-life in the blood (Supplemental Fig. 6D), and chronic experiments using RIPK2 inhibitor 1 agree with our preliminary pharmacokinetics, suggesting the efficacious quality of this inhibitor can last for up to 5-6 days after a $1-2-\mu \mathrm{g} / \mathrm{g}$ body weight intraperitoneal injection (data not shown). We are currently completing our pharmacokinetic analysis and will be exploring inhibition of intestinal inflammation in the $I L-10^{-/-}$model for inflammatory bowel disease, a model with spontaneous colonic inflammation by 8-10 weeks of age and an established model for IBD (Kuhn et al., 1993).

It has been demonstrated by several groups that RIPK2 has a unique requirement for NOD1 and NOD2 and functions in many pathways different from RIPK1, 3, or 4. Recently, Chirieleison et al. (2016) summarized the uniqueness of RIPK2 kinase domain within the RIPK family that could not be substituted for the kinase domain from RIPK1 or RIPK4. Indeed, RIPK2 inhibitors 1 and 2 did not inhibit RIPK1 biology nor ferroptosis, a form of cell death influenced by proteins of the RIPK family (Supplemental Fig. 6, A and B). As such, we believe that our identified RIPK2 inhibitors 1 and 2 can selectively modulate RIPK2-specific biology in agreement with the observations by Chirieleison et al. (2016).

Although we can observe a robust inhibition of autophosphorylation of RIPK2, $\mathrm{NF}_{\kappa} \mathrm{B}$, and alleviation of intestinal and airway inflammation injury in our mouse models of colitis and asthma, our kinome screen demonstrated in vitro inhibition of several other kinases, including 20\%-30\% inhibition of c-ABL, Aurora kinase $\mathrm{B}$, or ERBB2. We have demonstrated the importance of c-ABL in driving inflammation injury in our acute DSS model in the publication by Gordon et al. (2013). It is likely that our RIPK2 inhibitor 1 may interfere with the kinase activity of these off targets indirectly by resolving the inflammation. Many of these kinases are involved in growthrelated pathways, tumorigenesis pathways, and cell cycle control, such as Aurora kinase B (Borisa and Bhatt, 2017). These off-target effects may actually be beneficial in treating IBD, primary sclerosing cholangitis, primary sclerosing cholangitis/IBD-related CRC, and asthma, whereby a multitude of abnormal signaling involving inflammation, oxidative damage, DNA damage, and apoptotic abnormalities exists to manifest itself into a diseased state. Thus, eliminating all of these abnormalities using a single small molecule will promote a return to homeostasis and recovery from inflammation damage. It will be interesting to explore other inflammation states such as obesity, diabetes, cystic fibrosis, psoriasis, and arthritis to determine the importance of RIPK2 in driving inflammation damage and the usefulness of RIPK2 inhibitor 1 to alleviate the clinical symptoms associated with these diseases.

Comparison with Recently Identified RIPK2 Inhibitors. Recently, four inhibitors to RIPK2 were identified as OD36/OD38 (Tigno-Aranjuez et al., 2014) WEHI-435 (Nachbur et al., 2015), GSK-583 (Haile et al., 2016), and the one from Novartis (He et al., 2017). OD36 and OD38 were obtained through a small molecule macrocylization process from Oncodesign (Dijon Cedex, France) with $\mathrm{IC}_{50}$ values of $<100 \mathrm{nM}$ and the ability to interfere with MDP dependent RIPK2 activity. WEHI-435 was obtained by analysis of the RIPK2/ponatinib structure and the necrostatin-1/murine RIPK1 structure to obtain a structural face for the murine RIPK2 kinase domain (18-249) (Nachbur et al., 2015). Using this structural face, the computational biology used to obtain small molecules to associate with the RIPK2 ATP-binding pocket, GSK-583, was obtained using structural comparisons of RIPK2 with ponatinib. Last, the Novartis RIPK2 inhibitor was obtained in a similar manner to GSK583 and was based on a proprietary chemical library screen. Several hits were obtained, and after structural optimization, a RIPK2 inhibitor was obtained to inhibit RIPK2 kinase activity at $3 \mathrm{nM}(\mathrm{He}$ et al., 2017).

In this study, we used similarity-based virtual screening and molecular docking analysis to identify a RIPK2 inhibitor that can empirically inhibit the autophosphorylation of RIPK2, inhibit $\mathrm{NF}_{\kappa} \mathrm{B}$, and alleviate lung and intestinal inflammation efficacious at $1-2 \mu \mathrm{g} / \mathrm{g}$ body weight. RIPK2 inhibitors did not inhibit RIPK1 activity involved in ferroptosis or cell death nor have an effect on mitochondrial biology. In addition, RIPK2 inhibitor 1 appeared to also inhibit cell proliferation as determined by MTT assay, which did not appear to promote cell death. We find this curious and will be further exploring how RIPK2 inhibitors 1 and 2 can promote reduced proliferation and interfere with inflammation. Interestingly, RIPK2 has been demonstrated to be involved in the active growth of $\mathrm{CD} 90(+)$ intestinal stromal cells to suggest an "inflammatory" cross-talk between intestinal stromal cells and the epithelial cells (Owens et al., 2013), and we can observe robust activation of RIPK2 in IBD colon tissue sections immunostained with our proprietary pY474 RIPK2 antibody (Salla et al., unpublished observations). Inflammation signals from stromal cells and the epithelial cells will drive abnormal states to produce a cytokine storm that fuels malignant growth. Inhibitors to RIPK2 may have promising therapeutic potential to uniquely interfere with $\mathrm{NF}_{\kappa} \mathrm{B}$-dependent biology and offer an alternative to existing anti-inflammatory therapies.

\section{Acknowledgments}

The authors thank all the members of the Baksh laboratory for their helpful discussions.

\section{Authorship Contributions}

Conducted experiments: Salla, Aguayo-Ortiz, Ibrahim, Zare, Said, Blankstein, Fong, Manaloor, Pandya, Bhullar, Fiteh, Moore, Baksh. Participated in research design: Salla, Aguayo-Ortiz, Gibson, Goping, Velázquez-Martínez, Vliagoftis, Brocks, Hubbard, Baksh.

Performed data analysis: Salla, Aguayo-Ortiz, Hwang, Moore, Blankstein, Garcia, Meier, Baksh.

Wrote or contributed to the writing of the manuscript: Salla, Aguayo-Ortiz, Baksh.

\section{References}

Bernstein FC, Koetzle TF, Williams GJ, Meyer EF, Jr, Brice MD, Rodgers JR, Kennard O, Shimanouchi T, and Tasumi M (1977) The protein data bank: a computer-based archival file for macromolecular structures. J Mol Biol 112: $535-542$.

Borisa AC and Bhatt HG (2017) A comprehensive review on Aurora kinase: small molecule inhibitors and clinical trial studies. Eur J Med Chem 140:1-19.

Branquinho D, Freire P, and Sofia C (2016) NOD2 mutations and colorectal cancer Where do we stand? World J Gastrointest Surg 8:284-293.

Canning P, Ruan Q, Schwerd T, Hrdinka M, Maki JL, Saleh D, Suebsuwong C, Ray S, Brennan PE, Cuny GD, et al. (2015) Inflammatory signaling by NOD-RIPK2 is inhibited by clinically relevant type II kinase inhibitors. Chem Biol 22:1174-1184. 
Cao Y, Charisi A, Cheng LC, Jiang T, and Girke T (2008) ChemmineR: a compound mining framework for R. Bioinformatics 24:1733-1734.

Chirieleison SM, Kertesy SB, and Abbott DW (2016) Synthetic biology reveals the uniqueness of the RIP kinase domain. J Immunol 196:4291-4297.

Dai H, Ellis JL, Sinclair DA, and Hubbard BP (2016) Synthesis and assay of SIRT1activating compounds. Methods Enzymol 574:213-244.

Delaglio F, Grzesiek S, Vuister GW, Zhu G, Pfeifer J, and Bax A (1995) NMRPipe: a multidimensional spectral processing system based on UNIX pipes. J Biomol NMR 6:277-293

Dieleman LA, Palmen MJ, Akol H, Bloemena E, Peña AS, Meuwissen SG, and Van Rees EP (1998) Chronic experimental colitis induced by dextran sulphate sodium (DSS) is characterized by Th1 and Th2 cytokines. Clin Exp Immunol 114:385-391.

Dyson JK and Rutter MD (2012) Colorectal cancer in inflammatory bowel disease: what is the real magnitude of the risk? World J Gastroenterol 18:3839-3848.

Ermann J, Staton T, Glickman JN, de Waal Malefyt R, and Glimcher LH (2014) Nod/ Ripk2 signaling in dendritic cells activates IL-17A-secreting innate lymphoid cells and drives colitis in T-bet-/-.Rag2-/- (TRUC) mice. Proc Natl Acad Sci USA 111: E2559-E2566

Gonçalves P, Magro F, and Martel F (2015) Metabolic inflammation in inflammatory bowel disease: crosstalk between adipose tissue and bowel. Inflamm Bowel Dis 21: 453-467.

Gordon M, El-Kalla M, Zhao Y, Fiteih Y, Law J, Volodko N, Anwar-Mohamed A, ElKadi AO, Liu L, Odenbach J, et al. (2013) The tumor suppressor gene, RASSF1A, is essential for protection against inflammation -induced injury [published correction appears in PLoS One (2014) 9(1)]. PLoS One 8:e75483.

Haile PA, Votta BJ, Marquis RW, Bury MJ, Mehlmann JF, Singhaus R, Jr, Charnley AK, Lakdawala AS, Convery MA, Lipshutz DB, et al. (2016) The identification and pharmacological characterization of 6 -(tert-butylsulfonyl)-N-(5-fluoro- $1 \mathrm{H}$-indazol3-yl)quinolin-4-amine (GSK583), a highly potent and selective inhibitor of RIP2 kinase. J Med Chem 59:4867-4880.

Hayden MS and Ghosh S (2004) Signaling to NF-kappaB. Genes Dev 18:2195-2224. He X, Da Ros S, Nelson J, Zhu X, Jiang T, Okram B, Jiang S, Michellys PY, Iskandar M, Espinola S, et al. (2017) Identification of potent and selective RIPK2 inhibitors for the treatment of inflammatory diseases. ACS Med Chem Lett 8:1048-1053.

Jun JC, Cominelli F, and Abbott DW (2013) RIP2 activity in inflammatory disease and implications for novel therapeutics. J Leukoc Biol 94:927-932.

Kim S, Thiessen PA, Bolton EE, Chen J, Fu G, Gindulyte A, Han L, He J, He S, Shoemaker BA, et al. (2016) PubChem substance and compound databases. Nucleic Acids Res 44 (D1):D1202-D1213.

Kitzenberg D, Colgan SP, and Glover LE (2016) Creatine kinase in ischemic and inflammatory disorders. Clin Transl Med 5:31.

Koes DR and Camacho CJ (2012) Small-molecule inhibitor starting points learned from protein-protein interaction inhibitor structure. Bioinformatics 28:784-791.

Kühn R, Löhler J, Rennick D, Rajewsky K, and Müller W (1993) Interleukin-10 deficient mice develop chronic enterocolitis. Cell 75:263-274.

Kumar RK, Herbert C, and Foster PS (2008) The "classical" ovalbumin challenge model of asthma in mice. Curr Drug Targets 9:485-494.

Lagorce D, Sperandio O, Baell JB, Miteva MA, and Villoutreix BO (2015) FAFDrugs3: a web server for compound property calculation and chemical library design. Nucleic Acids Res 43 (W1):W200-W207.

Lasry A, Zinger A, and Ben-Neriah Y (2016) Inflammatory networks underlying colorectal cancer. Nat Immunol 17:230-240.

Lipinski CA, Lombardo F, Dominy BW, and Feeney PJ (2001) Experimental and computational approaches to estimate solubility and permeability in drug discovery and development settings. Adv Drug Deliv Rev 46:3-26.

Lupfer C, Thomas PG, Anand PK, Vogel P, Milasta S, Martinez J, Huang G, Green M, Kundu M, Chi H, et al. (2013) Receptor interacting protein kinase 2-mediated mitophagy regulates inflammasome activation during virus infection. Nat Immunol 14:480-488.

Lupfer CR, Anand PK, Liu Z, Stokes KL, Vogel P, Lamkanfi M, and Kanneganti TD (2014) Reactive oxygen species regulate caspase-11 expression and activation of the non-canonical NLRP3 inflammasome during enteric pathogen infection. PLoS Pathog 10:e1004410.

Madrid LV and Baldwin AS, Jr (2003) Regulation of NF-kappaB by oncoproteins and tumor suppressor proteins. Methods Mol Biol 223:523-532.
Madsen K, Cornish A, Soper P, McKaigney C, Jijon H, Yachimec C, Doyle J, Jewell L, and De Simone C (2001) Probiotic bacteria enhance murine and human intestinal epithelial barrier function. Gastroenterology 121:580-591.

Moriwaki K, Bertin J, Gough PJ, Orlowski GM, and Chan FK (2015) Differential roles of RIPK1 and RIPK3 in TNF-induced necroptosis and chemotherapeutic agent-induced cell death. Cell Death Dis 6:e1636.

Morris GM, Huey R, Lindstrom W, Sanner MF, Belew RK, Goodsell DS, and Olson AJ (2009) AutoDock4 and AutoDockTools4: automated docking with selective receptor flexibility. J Comput Chem 30:2785-2791.

Murgueitio MS, Rakers C, Frank A, and Wolber G (2017) Balancing inflammation: computational design of small-molecule toll-like receptor modulators. Trends Pharmacol Sci 38:155-168.

Nachbur U, Stafford CA, Bankovacki A, Zhan Y, Lindqvist LM, Fiil BK, Khakham Y, Ko HJ, Sandow JJ, Falk H, et al. (2015) A RIPK2 inhibitor delays NOD signalling events yet prevents inflammatory cytokine production. Nat Commun 6:6442.

Nakashima K, Hirota T, Suzuki Y, Akahoshi M, Shimizu M, Jodo A, Doi S, Fujita K Ebisawa M, Yoshihara S, et al. (2006) Association of the RIP2 gene with childhood atopic asthma. Allergol Int 55:77-83.

O’Boyle NM, Banck M, James CA, Morley C, Vandermeersch T, and Hutchison GR (2011) Open babel: an open chemical toolbox. J Cheminform 3:33.

Orlowski RZ and Baldwin AS, Jr (2002) NF-kappaB as a therapeutic target in cancer. Trends Mol Med 8:385-389.

Ostojic SM (2017) Mitochondria-targeted nutraceuticals in sports medicine: a new perspective. Res Sports Med 25:91-100.

Owens BM, Steevels TA, Dudek M, Walcott D, Sun MY, Mayer A, Allan P, and Simmons A (2013) CD90(+) stromal cells are non-professional innate immune effectors of the human colonic mucosa. Front Immunol 4:307.

Pettersen EF, Goddard TD, Huang CC, Couch GS, Greenblatt DM, Meng EC, and Ferrin TE (2004) UCSF Chimera-a visualization system for exploratory research and analysis. $J$ Comput Chem 25:1605-1612.

Sterling T and Irwin JJ (2015) ZINC 15--ligand discovery for everyone. J Chem Inf Model 55:2324-2337.

Suman S, Sharma PK, Rai G, Mishra S, Arora D, Gupta P, and Shukla Y (2016) Current perspectives of molecular pathways involved in chronic inflammationmediated breast cancer. Biochem Biophys Res Commun 472:401-409.

Tang BL (2016) Sirt1 and the mitochondria. Mol Cells 39:87-95.

Tigno-Aranjuez JT, Asara JM, and Abbott DW (2010) Inhibition of RIP2's tyrosine kinase activity limits NOD2-driven cytokine responses. Genes Dev 24:2666-2677.

Tigno-Aranjuez JT, Benderitter P, Rombouts F, Deroose F, Bai X, Mattioli B, Com inelli F, Pizarro TT, Hoflack J, and Abbott DW (2014) In vivo inhibition of RIPK2 kinase alleviates inflammatory disease. J Biol Chem 289:29651-29664.

Trott O and Olson AJ (2010) AutoDock Vina: improving the speed and accuracy of docking with a new scoring function, efficient optimization, and multithreading. $J$ Comput Chem 31:455-461.

Vanden Berghe T, Hassannia B, and Vandenabeele P (2016) An outline of necrosome triggers. Cell Mol Life Sci 73:2137-2152.

Vieira SM, Cunha TM, França RF, Pinto LG, Talbot J, Turato WM, Lemos HP, Lima JB, Verri WA, Jr, Almeida SC, et al. (2012) Joint NOD2/RIPK2 signaling regulates IL-17 axis and contributes to the development of experimental arthritis. J Immunol 188:5116-5122. Vriend G (1990) WHAT IF: a molecular modeling and drug design program. J Mol Graph 8:52-56, 29 .

Williamson KD and Chapman RW (2015) Primary sclerosing cholangitis: a clinical update. Br Med Bull 114:53-64.

Wu S, Kanda T, Nakamoto S, Imazeki F, and Yokosuka O (2012) Knockdown of receptor-interacting serine/threonine protein kinase-2 (RIPK2) affects EMTassociated gene expression in human hepatoma cells. Anticancer Res 32: 3775-3783.

Address correspondence to: Dr. Shairaz Baksh, Division of Hematology/Oncology, Department of Pediatrics, Faculty of Medicine and Dentistry, 3-020E Katz Group Centre for Pharmacy and Health Research, 113 Street 87 Avenue, University of Alberta, Edmonton, AB T6G 2E1, Canada. E-mail: sbaksh@ualberta.ca 